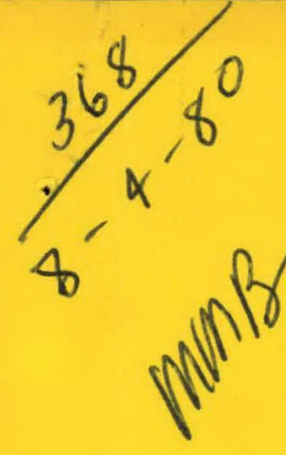

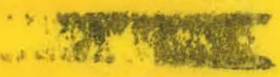

DOE/NASA TM-78286

DOE/NASA TECHNICAL MEMORANDUM

\title{
ASHMET - A COMPUTER CODE FOR ESTIMATING INSOLATION INCIDENT ON TILTED SURFACES
}

By Robert F. Elkin and Ronald G. Toelle

Systems Analysis and Integration Laboratory

National Aeronautics and Space Administration

George C. Marshall Space Flight Center, Alabama 35812

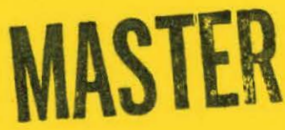

May 1980

For the U. S. Deparment of Energy

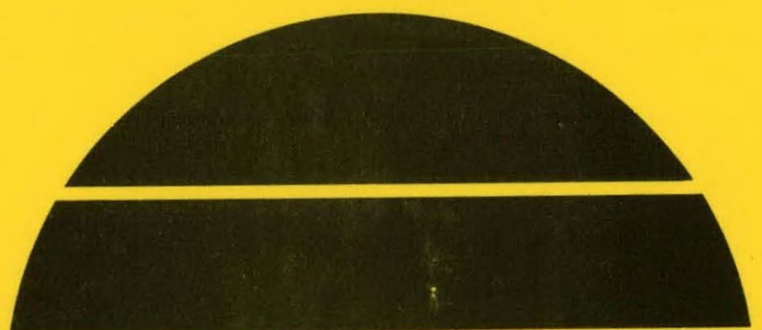

\section{U.S. Department of Energy}




\section{DISCLAIMER}

This report was prepared as an account of work sponsored by an agency of the United States Government. Neither the United States Government nor any agency Thereof, nor any of their employees, makes any warranty, express or implied, or assumes any legal liability or responsibility for the accuracy, completeness, or usefulness of any information, apparatus, product, or process disclosed, or represents that its use would not infringe privately owned rights. Reference herein to any specific commercial product, process, or service by trade name, trademark, manufacturer, or otherwise does not necessarily constitute or imply its endorsement, recommendation, or favoring by the United States Government or any agency thereof. The views and opinions of authors expressed herein do not necessarily state or reflect those of the United States Government or any agency thereof. 


\section{DISCLAIMER}

Portions of this document may be illegible in electronic image products. Images are produced from the best available original document. 
TECHNICAL REPORT STANDARD TITLE PAGE

\begin{tabular}{|c|c|}
\hline $\begin{array}{l}\text { 1. REPORT NO. } \\
\text { DOE/NASA TM-78286 }\end{array}$ & 3. RECIPIENT'S CATALOG NO. \\
\hline \multirow{2}{*}{$\begin{array}{l}\text { 4. TITLE AND SUBTITLE } \\
\text { ASHMET - A Computer Code for Estimating Insolation } \\
\text { Incident on Tilted Surfaces }\end{array}$} & $\begin{array}{l}\text { 5. REPORT DATE } \\
\text { May } 1980\end{array}$ \\
\hline & 6. PERFORMING ORGANIZATION CCIDE \\
\hline $\begin{array}{l}\text { 7. AUTHOR(S) } \\
\text { Robert F. Elkin and Ronald.G. Toelle }\end{array}$ & 8. PERFORMING ORGANIZATION REPORT \\
\hline \multirow{3}{*}{$\begin{array}{l}\text { 9. PERF ORMING ORGANIZATION NAME AND ADDRESS } \\
\text { George C. Marshall Space Flight Center } \\
\text { Marshall Space Flight Center, Alabama } 35812\end{array}$} & 10. WORK UNIT. NO. \\
\hline & 11. CONTRACT OR GRANT NO. \\
\hline & 13. TYPE OF REPOR' $\&$ PERIOD COVERE \\
\hline \multirow{2}{*}{$\begin{array}{l}\text { 12. SPONSORING AGENCY NAME AND ADORESS } \\
\text { National Aeronautics and Space Administration } \\
\text { Washington, DC } 20546\end{array}$} & Technical Memorandum \\
\hline & 1.1. SPONSORING AGENCY CODE \\
\hline
\end{tabular}

15. SUPPLEMENTARY NOTES

Prepared by Systems Analysis and Integration Laboratory.

16. ABSTRACT

A computer code, ASHMET, has been developed by MSFC to estimate the amount of solar insolation incident on the surfaces of solar collectors. Both tracking and fixed-position collectors have been included. Climatological data for $248 \mathrm{U}$. S. locations are built into the code. This report describes the methodology of the code, and its input and output.

The basic methodology used by ASHMET is the ASHRAE clear-day insolation relationships modified by a clearness index derived from SOLMET-measured solar radiation data to a horizontal surface. 


\section{ACKNOWLEDGMENT':}

The authors wish to thank all those at MSIC who contributed to this report through reviewing and making many valuable suggestions.

Particular thanks is given to Harrell Phillips, Flight Mechanics Branch, SA\&I Laboratory, for his assistance in the development of the generalized methodology for determining the incidence angle the sun vector forms with the surface normal of rotating collectors. 
TABLE OF CONTENTS

\begin{tabular}{|c|c|c|}
\hline & & \\
\hline I. . & INTRODUCTION & 1 \\
\hline II. & ASHMET I METHODOLOGY & 13 \\
\hline & A. Total Insolation to a Fixed-Position Collector & 3 \\
\hline & B. Total Insolation to a Munthly T1lt Adjusted Collector & 4 \\
\hline & C. Beam Tracking Collector & 4 \\
\hline & $\begin{array}{l}\text { D. Direct Insolation to a Fixed Aximuth Tracker } \\
\text { Rotating About its } \Lambda \text { xis }\end{array}$ & 5 \\
\hline & $\begin{array}{l}\text { E. Direct Insolation to Fixed-Position and } \\
\text { Monthly Tilt Adjusted Flat Plate Collectors }\end{array}$ & 6 \\
\hline III. & ASHMET II METHODOLOGY & 7 \\
\hline & REFERENCESS & 9 \\
\hline & NOMENCLATURE & 10 \\
\hline & APPENDIX A - ASHMET INPUT AND OUTPUT & 11 \\
\hline & APPENDIX B - ASHMET SOURCE LISTING & 12 \\
\hline
\end{tabular}

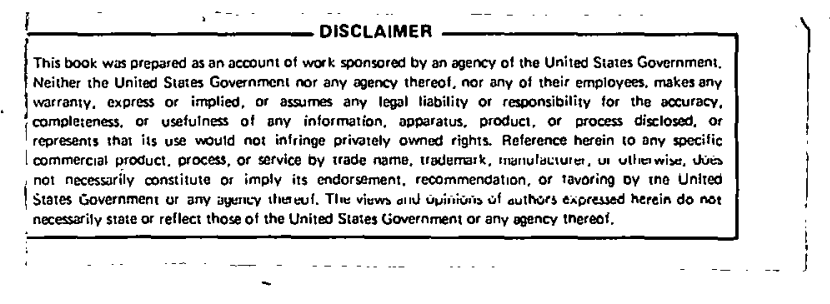

iii 
SUMMARY

A computer code has been developed to estimate the amount of solar insolation indident on the surfaces of several types of collectors. Both tracking and fixed position collectors have been included. Climatological data for 248 U.S. locations are built into the code. This report describes the methodology of the code, its input and output.

\section{INTRODUCTION}

In designing or analyzing a solar system, the principle question to be answered is how much of the available solar energy can the system deliver to loads. This fraction of available energy is dependent on several parameters: amount of solar energy available, type of collectors used, efficiency of system heat exchangers, system losses, etc. The primary driver is, of course, the solar energy available (energy incident on the surface of the collector array) to the system. This report describes a computer code, ASHMET, developed by MSFC to analytically estimate the solar energy incident on the collector array surface cover.

Two verstons of ASHMET exist. Both versions utilize the same basic methodology: ASHRAE relationships [1] are used to obtain clear day total daily insolation incident on the collector surface for a representative day (defined in ASHMET as the 2lst day of the month) of each month of the year; the clear day total (direct + diffuse + reflected) daily insolation is then multiplied by a clearness index to obtain the typical or average daily insolation. The clearness index was derived from SOLMET measured insolation [2] and the ASHRAE clear day insolation. In reference 2 average daily total insolation on a horizontal surface for 248 U.S. locations are given for each of the 12 months of the year. These data were divided by the ASHRAE clear day insolation at the same geographical location and the resulting tables built into ASHMET as clearness index. The assumption is made here that the clearness index does not change with collector tilt angle or azimuthal orientation.

Major differences between the two versions are in the procedures for breakout of direct and diffuse insolation. Version I uses the ASHRAE relationships of reference 1; Version II the correlation of Liu and Jordan [3]. Version II resulted when comparisons of incident radiation to tilted surfaces obtained using the ASHRAE relationships for separation of direct and diffuse components to similar data obtained using the LIu and Jordan correlation indicated significant differences in the two sets of data for some geographic locations. Since, at present, insufficient data is available to verify either of the methods, and since the Liu and Jordan procedure is widely used in the solar industry, it was decided to add the Liu and Jordan methodology to ASHMET as an option. 
Both versions have the capabilities of calculating incident solar radiation on the collector surface for six types of collectors:

(1) Fixed position flat plate collector - total insolation

(2) Monthly tilt adjusted flat plate collector - total insolation

(3) Beam tracking collector (sun tracker - direct insolation only)

(4) Fixed azimuth tracker - direct insolation only (tracks in plane of tilt, rotating about tilt axis)

(5) Fixed position flat plate collector - direct insolation

(6) Monthly tilt adjusted flat plate collector - direct insolation only.

In type (2) and (6) the monthly tilt angle adjustment is accomplished by setting the tilt equal. to latitude minus declination using the decilination angle for the 21 st day of the month. Additionally, Vereion II of ASHMET has a type (7), which allows calculation of direct insolation to a collector rotating about its east-west axis. The angle of rotation is chosen such that the angle of incidence between the sun vector and the normal to the collector surface is minimized. (NOTE: Type (4) of both can be used to obtain the same results as type (7) of Version II with some judicious manipulation of input data. However, for the casual user of ASHMET it was felt that a straightforward option for this specific type of collector would be less confusing. Also, the methodology is greatly simplified, reducing computer time.)

The following two sections give the details of methodology used in ASHMET: Input and output for the code are described in Appendix $A$ and computer 1istings of ASHMET $I$ and ASIMET II in Appendix B. 
II. ASHMET I METHODOLOGY

A. Tota1. Insolation to a Fixed Position Collector

Clear day hourly total insolation to a tilted surface are obtained from the ASHRAE relationships [2]:

$$
\begin{aligned}
h \text { TOTAL, CLR DAY }= & \frac{(\mathrm{A}) \cos \theta}{\mathrm{e}^{B / \sin \beta}}+\frac{(\mathrm{A})(\mathrm{C}) \cos \theta}{\mathrm{e}^{B / \sin \beta}} \cdot\left(\frac{1+\cos S}{2}\right) \\
& +\left(\frac{\mathrm{A}}{\mathrm{e}^{B / \sin \beta}}\right)(\sin \beta+\mathrm{C})(\rho)\left(\frac{1-\cos S}{2}\right)
\end{aligned}
$$

Where: $A, B$, and $C$ are monthly varying coefficients taken from Table 1 in chapter 22 of reference 1 .

$\theta$ is the angle of incidence the sun's rays form with the collector surface (relative to the surface normal) defined by

$$
\begin{aligned}
\cos \theta & =\cos S \sin \beta+\sin S \cos \gamma \tan L \sin \beta \\
& -\sin S \cos \gamma \sin \delta / \cos L+\sin S \sin \gamma \cos \delta \sin \omega .
\end{aligned}
$$

$\beta$ is the solar altitude (angle between direction of sun and local horizontal) given by

$$
\sin \beta=\cos L \cos \delta \cos \omega+\sin L \sin \delta
$$

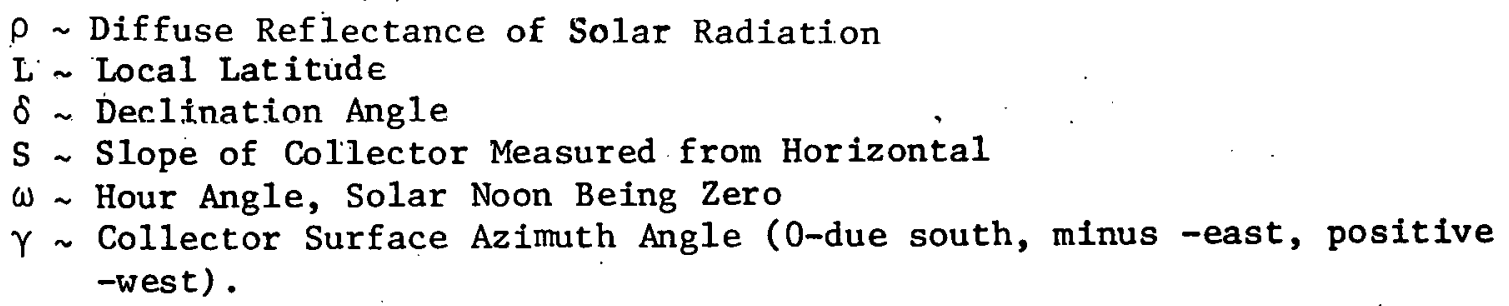

In equation (1) the first term on the right hand side represents the direct insolation received, the second term the diffuse and the third the reflected component. The declination angle, $\delta$, is obtained from a table [1] for the 21st day of each month.

Using equation (1) hourly insolation distribution incident on the tilted surface is calculated for the 21 st day of each month of the year and summed for the day:

H TOTAL, CLR DAY $=\int_{t=\text { S.R. HOUR }}^{\text {S.S. HOUR }}$ (h TOTAL, CLR DAY) $d t \approx \sum_{6 \text { A.M. }}^{6 \text { P.M. }}$ h TOTAL, CLR DAY 
The clear day daily total is then multiplied by a clearness index to obtain a typical day's insolation for each month. The clearness index was derived from the SOLMET insolation data (to a horizontal surface) of reference 2: mean SOLMET daily horizontal total insolation for each month of the year was divided by the clear day total horizontal insolation calculated by equation (4) for each of the geographic locations presented in reference 2. These data were incorporated in the ASHMET program. It is assumed that the clearness indexes derived for horizontal data are applicable to tilted surfaces.

\section{B. Total Insolation to a Monthly Tilt Adjusted Collector}

On any given day the daily direct insolation incident on a fixed position collector will be maximized if the collector tilt angle is set equal to the latitude minus the declination. ASHMET, therefore, has an option for setting the collector tilt angle equal to the desired site latitude minus the declination angle for the $21 \mathrm{st}$ day of each month. Once the tilt angle is calculated the program proceeds as for the total insolation to a fixed position collector option doecribed previnusly.

\section{Beam Tracking Collector}

This option provides the direct insolation incident on the surface plane of a sun tracking collector. Clear day direct insolation (for incidence angle $\theta=0^{\circ}$ ) is obtained from the ASHRAE equation:

$$
H_{\text {BEAM, CLR DAY }=}\left[\begin{array}{c}
\text { SUNSET HR. } \\
{\left[A / \mathrm{e}^{\mathrm{B} / \sin \beta}\right] \mathrm{dt}} \\
\mathrm{t}=\text { SUNRISE HR. }
\end{array}\right.
$$

Typical daily beam insolation (for the 21 st day of each month) is calculated by multiplying the clear day value by the average daily percent sunshine (\% SS) for that month and the location desired. The percent sunshine was derived as follows.

It is assumed, as suggested by several authors, that the typical daily insolation can be represented by

$$
\begin{aligned}
& H_{\text {TOTAL }}=[\mathrm{F}] \text { [H TOTAL, CLR DAY] } \\
& F=c[a+b(\% S S)]
\end{aligned}
$$

The coefficients $a, b$, and $c$ were determined from the SOLMET insolation data and the annual mean daily percent sunshine data of reference 4 . As expected the coefficients varied from location to location within the United States. 
However, areas of constant coefficients could be determined from the data. These areas are delineated in Figure 1 along with the values of $a, b$, and $c$ for each area.

Equation (6) is now solved for the percent sunshine:

$$
\% \text { SS }=\left[\left(\frac{\text { HTOTAL }_{\text {HOTAL, CLR DAY }}}{\text { HOT }_{\text {TO }}}\right)-\mathbf{a}\right] / \mathrm{b}
$$

In equation ( 7 ) the term $\mathrm{H}_{\text {TOTAL }} / \mathrm{H}_{\text {TOTAL, CLRDAY }}$ is the previously discussed clearness index derived from the SOLMET data.

The use of equation (7) to calculate the average daily percent sunshine circumvents two problem areas. First, the necessity of adding a second table of data to the program (monthly average daily percent sunshine for 248 geographic locations) is avoided. Secondly, the sunshine data of reference 4 covers only one-fourth of the 248 locations for which insolation data is available. Thus considerable extrapolation of the sunshine data would be necessary if all the SOLMET sites were to be included. It was felt a more accurate approach would be to define areas of constant coefficients $a, b$, and $c$ of equation (6) from the sunshine data of reference 4 and then use the SOLMET insolation data per equation (7) to define percent sunshine for locations not covered by reference 4 .

D. Direct Insolation to a Fixed Azimuth Tracker Rotating About its Axis

With this option direct insolation to the surface of a collector rotating about its axis is calculated. Orientation of axis is determined by specifying its azimuth and tilt angles (input by program user).

Similar to the previously discussed collector systems the daily distribution of clear air hourly direct insolation for the typical, day of each month is first calculated from the ASHRAE equation:

${ }^{h_{D I R E C T}}$ CLR DAY $=\frac{A \cos \theta}{\mathrm{e}^{B / \sin \beta}}$

WHERE: $\quad \cos O=\sqrt{1.0-\left[\sin \beta \sin S-\cos \beta \cos S \cos \left(A Z_{s}+A Z\right)\right]^{2}}$ 
$\mathrm{Az}$ is the collector longitudinal axis azimuth angle measured from due south with east negative and west positive. $\Lambda z_{s}$ is the solar azimuth angle and is obtained from:

$$
A Z_{s}=\sin ^{-1}\left[\frac{\cos \delta \sin \omega}{\cos \beta}\right]
$$

The typical daily direct insolation for each month is now obtained by multiplying the clear day insolation by the percent sunshine term described in the previous section on beam trackers.

E. Direct Insolation to Fixed Position and Monthly Tilt Adjusted Flat Plate Collectors

Calculations for these systems proceed the same as for the calculation of the total insolation incident on their surfaces with one exception: typical daily incident insolacton is ul tained by multiplying the ASHRAE clear day direct radiation by the percent sunshine term of subsection $B$ rather than the clearness index of subsection $A$. 
The basic ASHMET II methodology is the same as that of ASHMET I with the exception of the breakout of horizontal diffuse and direct insolation for fixed position collectors (including those with monthly tilt adjustment). For these collector systems the breakout of horizontal diffuse and direct insolation is taken from the correlation of Liu and Jordan [3] instead of the ASHRAE methodology. Calculations of incident insolation for beam. tracking and azimuth tracking surfaces remains unchanged.

From the Liu and Jordan correlation clear day diffuse insolation to a horizontal surface is:

$$
\begin{aligned}
& \left(\frac{h_{\text {DIFF }}}{h_{\text {TOTAL }}}\right)_{\text {HRLY, HORIZ, CLR. DAY }} \\
& \qquad=1.39-4.027\left(K_{T}\right)+5,531\left(K_{T}\right)^{2}-3.108\left(K_{T}\right)^{3}
\end{aligned}
$$

Where:

$$
K_{T}=\left(\frac{H_{T O T A L}}{H_{\text {EXTRATERRESTRIAL }}}\right) \text { DAILY, HORIZ }
$$

The clear day direct insolation component is then:

$$
\begin{aligned}
& \text { ('hDIR) HRLY, HORIZ = (hTOTAL) HRLY, HORIZ, CLR DAY } \\
& -\left[\left(\frac{\text { hDIFF }}{\text { hTOTAL }}\right)(\text { hTOTAL })\right] \text { HRLY, HORIZ, CLR DAY }
\end{aligned}
$$

Total clear day hourly to a tilted surface is obtained as in the ASHMET I program:

$$
\begin{aligned}
& \left(h_{\text {TOTAL }}\right)_{\text {HRLY, CLR DAY }}=\left(h_{\text {DIR }}\right)_{\text {HRLY, HORIZ }} \cos \theta \\
& +\left(h_{D I R}+h_{D I F F}\right)_{\text {HRLY, HORIZ }}\left(\frac{1-\cos S}{2}\right) \\
& +(\text { hDIFF })_{\text {HRLY, HORIZ }}\left(\frac{1+\cos S}{2}\right)
\end{aligned}
$$

Calculation of the typical insolation incident on tilted surface for each month of the year then proceeds as in ASHMET I: typical total insolation by multiplying the clear day daily total to the tilted surface by the appropriate clearness index; typical direct insolation by applying the percent sunshine term to the clear day direct insolation component. 
Additionally, ASHMET II has a seventh collector type option: direct insolation incident on a horizontal surface rotating about an east-west axis in a northsouth direction. For this case the clear day daily direct component is (for the 21st day. of the month):

$$
H_{\text {DIR, CLR DAY }}=\int_{\substack{\text { SUNSET HR. } \\\left[A / e^{B / \sin \beta}\right]\left[1-(\cos \delta \cos \omega)^{2}\right]^{1 / 2} d t}}^{\text {SUNRISE HR. }}
$$

(INOTE: The previously discussed option of a fixed azimuth tracker rotating about its axis may also be used to obtain incident insolation for the case. However, the casual user of ASHMET may not be sufficiently knowledgable of the program methodology to use the fixed azimuth tracker option in this manner; also, computer time will be saved by using equation (14).) 
1. ASHRAE Handbook of Fundamentals, 1972, Chapter 22.

2. Cinquemani, V., Owenby, J. R. and Baldwin, R. G., Input Data for Solar Systems, U.S. Department of Commerce, November 1978.

3. Liu, B. Y. H. and Jordan, R. C., The Interrelationship and Characteristic Distrłbution of Direct, Diffuse and Total Solar Radiation, Solar Energy IV (3), July 1960.

4. Solar Heating Systems Design Manual, International Telephone and Telegraph Corporation Bulletin TESE-576. 


\section{NOMENCLATURE}

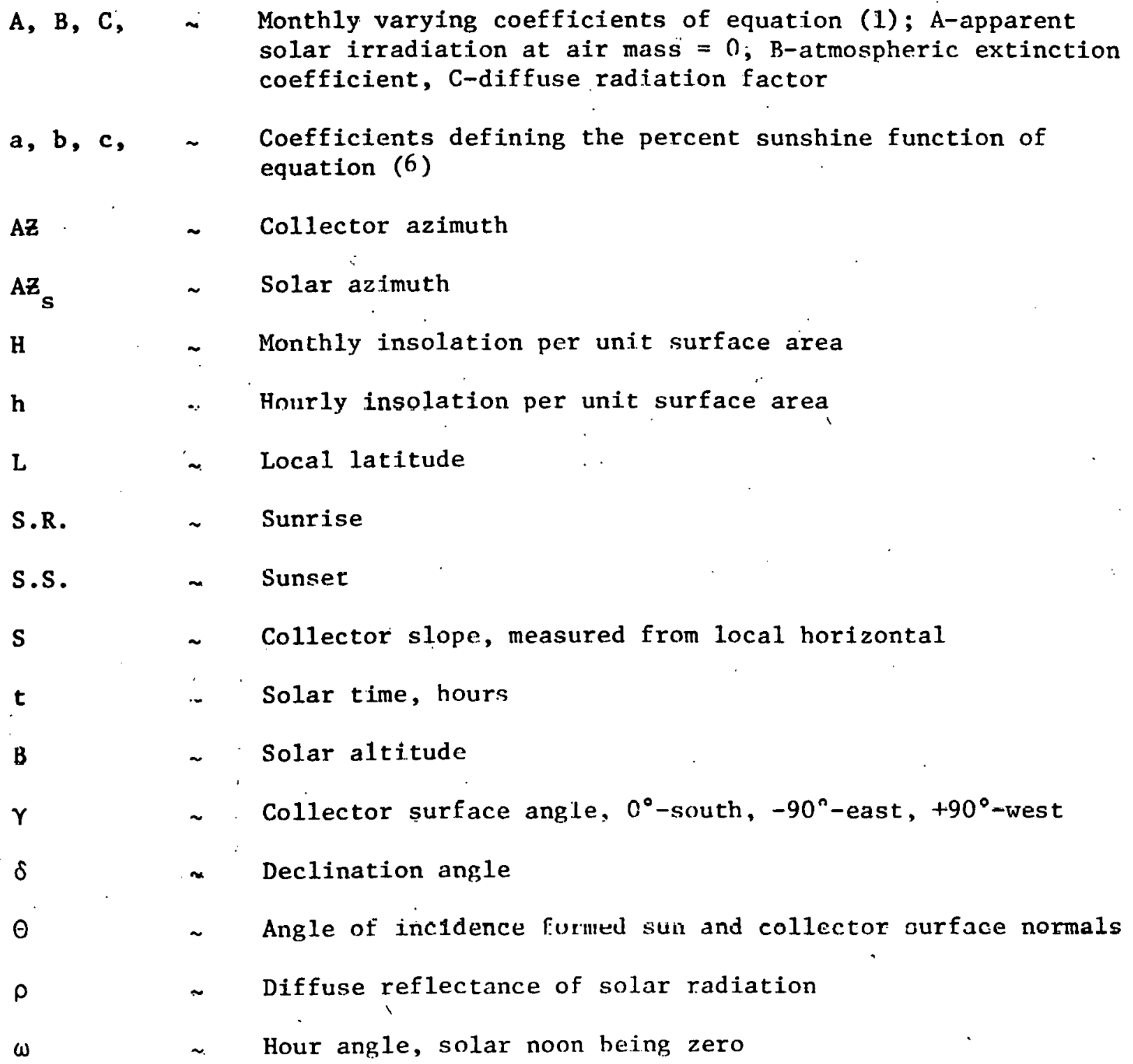


APPENDIX A

ASHMET TNPUT

ASHMET is programmed for interactive use with a computer and a remote terminal. Once the program is started the user will find ASHMET self-explanatory. Questions concerning program options are asked and input parameters are called for with explanations of the parameters. A sample input case is shown in Figure 2.

The input data consists of

1. Latitude of desired location (format - F9.4)

2. Slope of collector measured from the horizontal (format - F9.4)

3. Azimuth of collector surface $\left(0^{\circ}\right.$ south, minus-easterly facing, pluswesterly facing) (format - F9.4)

4. Type of collector-beam tracker, fixed position flat plate, etc. (Program informs the user of code number to be used for each type.)

5. Ground reflectance (if desired)

6. City location number from Tahle 1.

If city location desired is not listed in the table the user has the option of inputing twelve monthly values of clearness indexes (ratio of monthly typical day's total insolation to clear day insolation in percent) in the format 12F4.0.

\section{ASHMET OUTPUT}

A sample output is shown in Figure 3 and should be self-explanatory. The type of collector system called for is.output along with the other required input data (latitude, slope, etc.). Monthly clearness indexes will be output only if they are input by the user. Insolation output includes the hourly clear day incident insolation, clear day and typical daily insolation for each month. 


\section{APPENDIX B}

ASHET 1

DIMENSION A(12),D(12),C(12), DEC 12), REFLT(12),DLYSUM(12),

IDAYS (12), TIME (13), BTA(13), SLRAZ (13), HRLYRD (13,12), DIFRAD(13)

DINENSION BRAD(13), DIRRAD(13), HORZRD (13), REFRO (13)

DIMENSION DECL(12), TIILT(12)

DIAENSION PCTSS(12), DLPRSH(12)

C REAL LAT CONSTAMT DATA

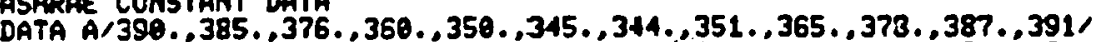

DATA B/.142, .144,.156,.18,.196, .205,.247,.201,.177,.16,.149,.142/

DATA C/.058,.06,.071,.097,.121,.134,.136,..12,,.092,.073,.063,.057

DATA DEC $-20 .,-10.8,6 ., 11.6,20 ., 23.45,20.6,12.3,0 .,-10.5,-19.8$,

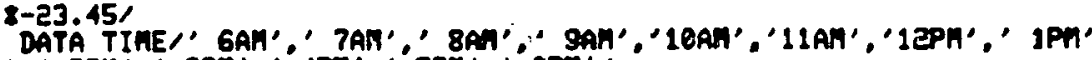

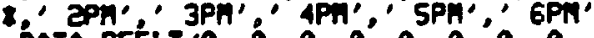

0. 0.00 .0 .0 .0 .0 .0

DATA DAYS/31..,28.,3i..30.,31.,30.,31.,31.,30.,31.,30.,31.1

DATA N 'NN'

DTR=57.29578

OSS FOPMAT IX, 'DEFINE OUTPUT DEUICE"/ INPUT I FOR TERMINAL OR'

' INPUT 6 ' FOR LINE PRINTER')

OPEN(UNIT-6, NAFE.'PPRINT. LST', TYPE= 'NEL'

C READ DATA FOR SITE

1 WePTE(1,2)

GORMAT (IX, 'INPUT LATITUDE DEG')

3 FORDAT 3 LAT

3 FoRMAT(F9.4)

4 FORHATI IX.' 'INPUT TILT ANGLE FRON HORIZONTAL DEG')

READ (1,3iTILT

URITE $(i, 5)$

5 FORATT(IX, INPUT AZIWUTH AHGLE DEG O DUE SOUTH + LEST - EAST') READ(1,3)AZI

555 FORHAT (IX,'IS GROUND REFLECTANCE DESIRED? (Y OR N)') READ (1,9)IR If (IR.EO.NIOO TO 80

URITE(1,6)

6 FOHAT(1X, 'PRELOADED UALUES OF GROUND RETLECTANCE ARE')

thITe (1,7) REF (T(I),I-1,12)

7 Fogmar(ix, 12r3.2)

URITE $(1,8)$

8 FORPAT (iX.'IS CHANGE DESIRED? (Y OR NM')

- Foditília

IFIIA.EQ.Nं, CO To 84 


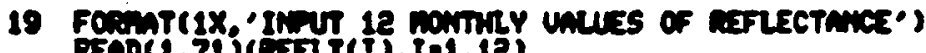

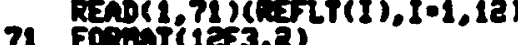

Co ro 84

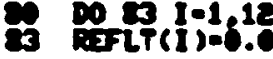

84 FolTE(2 81 '

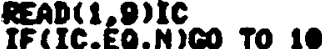

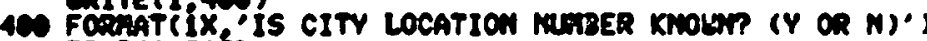
IF(IL.EO.Hico to 2

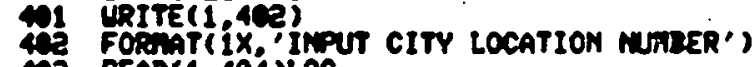
13 READC1,404)LOC

CALL SUHSHN(LOC,PCTSS, KSSN)

$c$

o) ERTE(1,91)

年 romat (1X, 12r4.0) nITE(1, g2) (PCTsS(I), I-1,12) Cint $(18)$ IFIIA.EO.NiCO TO 10

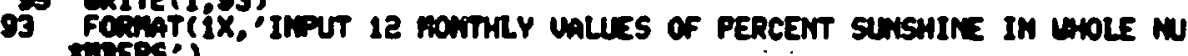

a)

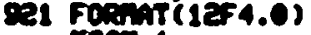

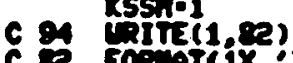

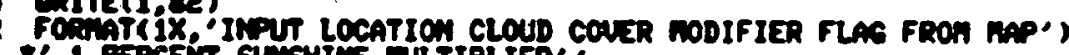

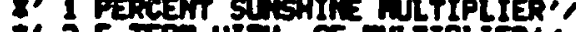

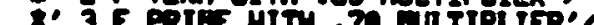

Co $f$ or

* 5 clesar är Insolation')

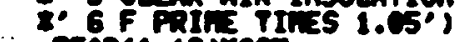

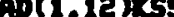

11 Trtis(i)

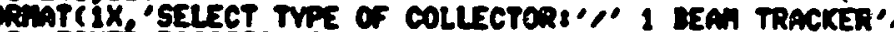

*' 2 FIXID POSITION FLAT PLATE UITH TOTAL IUSOLATION'

8' 3 AZIIMm TACEKER - DIRECT IHSOLATION OMLY'

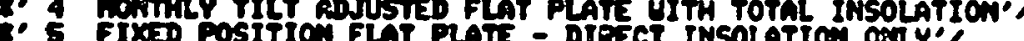

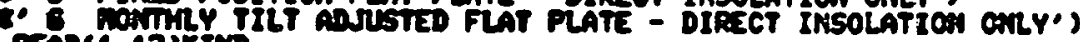

18

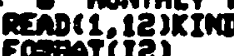

c cond 20.6

pos jol.18 
22 prysun(1)-o.

C SET LP IRIG CONSTANTS FOR IAPUT MUALERS

LAT-1070

Lar

TLAT-SLAT/CLAT

ctit=cos(TIt)

Xox-.5*(1.-CTLT)

c

Do $1000 N=1,12$

ITIคต

REFHPY $X X X X R E F L T(n)$

DECL(R)=DEC(A)/DTR

SDECL-SI

CDECL=COS(DECL(A))

C DAlLY LOOP FOR 6 AM TO 6PM

DO $5501 T m_{0}-6,6,1$

ITiR:1TI $A+1$

RQHGL: 15 . BFLOAT (ITH)

实

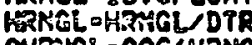

CHRTHGL - cos (HaNGL

SHRTHOL =SIN (HANGL)

SBTA.CATECLCLPCHRGL+SLATZSCECL

IF(

20 BTA(ITAT) $01251,51,20$

CBTa=cos(gTa(rom)

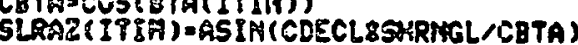

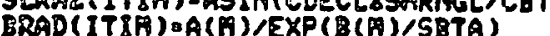

GO PO $(25,50,75,100,125,180)$ KIND

C

25 HRLYRD(ITIM,M) - BRAD (ITIF)

$c$

$60 \mathrm{TO} 498$

FLAT PLATE

50 TALAPY-CTLFZSBTA4STL IZ (CAZZ((TLATZSBTA)-!SJECL/CLAT))+SAZ ZCDECL

51 IF(TALTipy $) 51,51,52$

DIPRap (ITIM): $=0$.

IFraD (19IM).e.

CO 1053

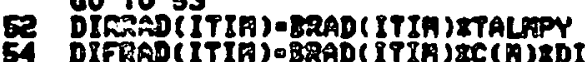

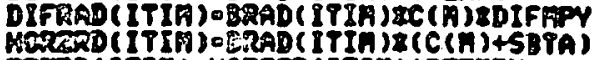

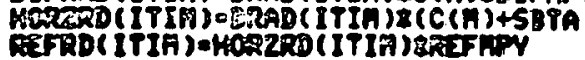




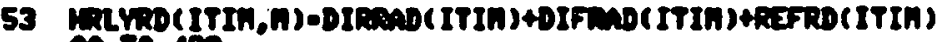

c. Rotatino azimutr tanckino collecton

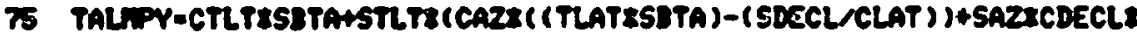

15M.ive()

76 IF (TALLY) $51,51,75$

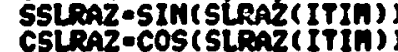

Se-CBTAXSSLRAZ

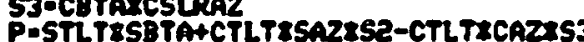

UL-SBTA-PISTLT

20-Sz-prCTLT2SAZ

U3--(S3+P\&CTL TXCAZ)

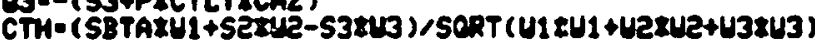

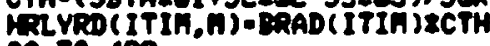

MONTHLY TILT ADJUSTED

CALCULATE TIIT FBOA LAT-DECL

100 IILT-LAT-DECL(

TIILT(n)-TILT

crtocos(tit

IF(KIND.EQ.6) GO TO 125

$110 \times \times \times=.5 \pm(1 .-$ CTLT $)$

DIFMovel $-x \times x$

er

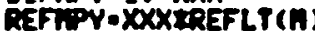

c

To 50

125

EI DIFUSE AND REFLCTED TO ZERO FOR FLAT PLATE DIRECT ONLY

Difhiryo.

c

sur 502 D.

499 DLrsun(n)

Yoxs

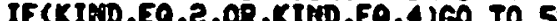

CO TO (581, 582,583, 584, 585,586 587) Ks

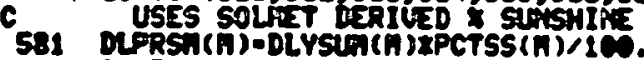

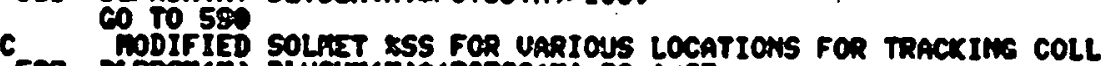

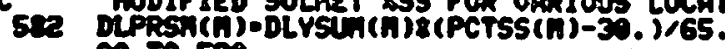

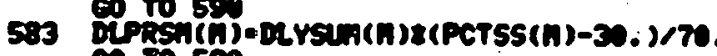

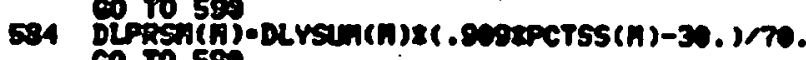

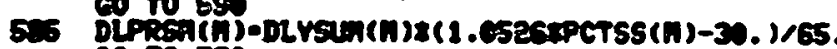

ses ofrasn(

ses co.To Eg .

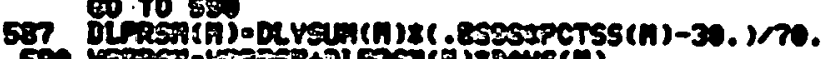


$\because$

trofity

lne intr(I)

0 To (501,502,503,504,505, 505)kIn

501 Mituritisis)

a. Fo.

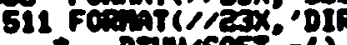

i-jorth

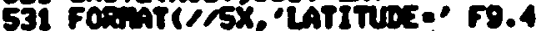

ene

(nIT) (now. 512)

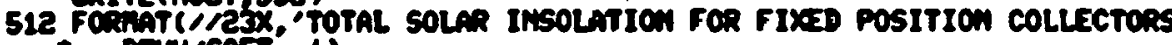
- - BTH:ACT - ')

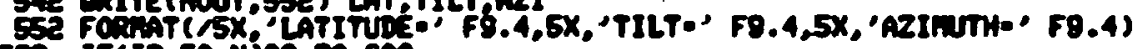

ses If IR.EO.Nieo to E.

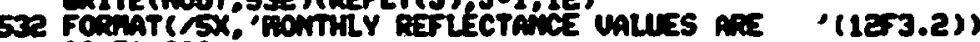

60 ro 69.

503 Uite(tiour. 513 )

513 Fo.

523 CRITE(noUT, SE2) LAT, IILT, A21

60 To 60

5NA UITE(NoN.514)

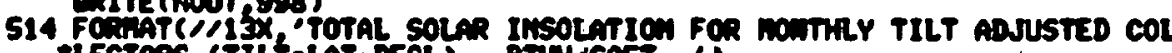
LCTOCS (TILT-LAT-LECL) - BTUWSOTT -

5a (n)

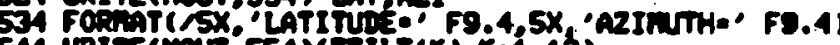

-54 Fonan

$75275,3 \times 5510$

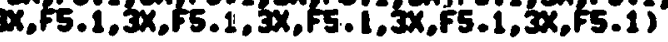

Ex

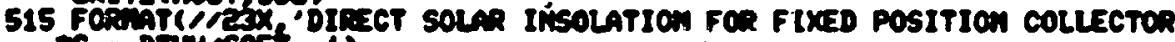

IS - BTwasert -

eo to sas.

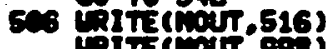

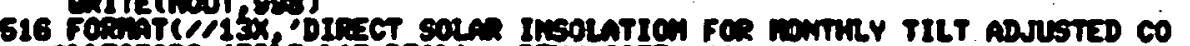

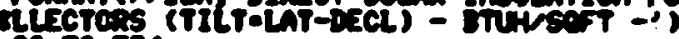

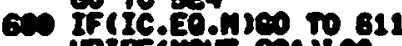

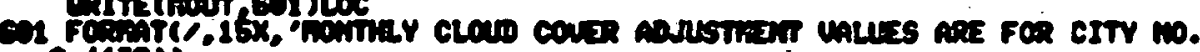

028 inturimentoser 


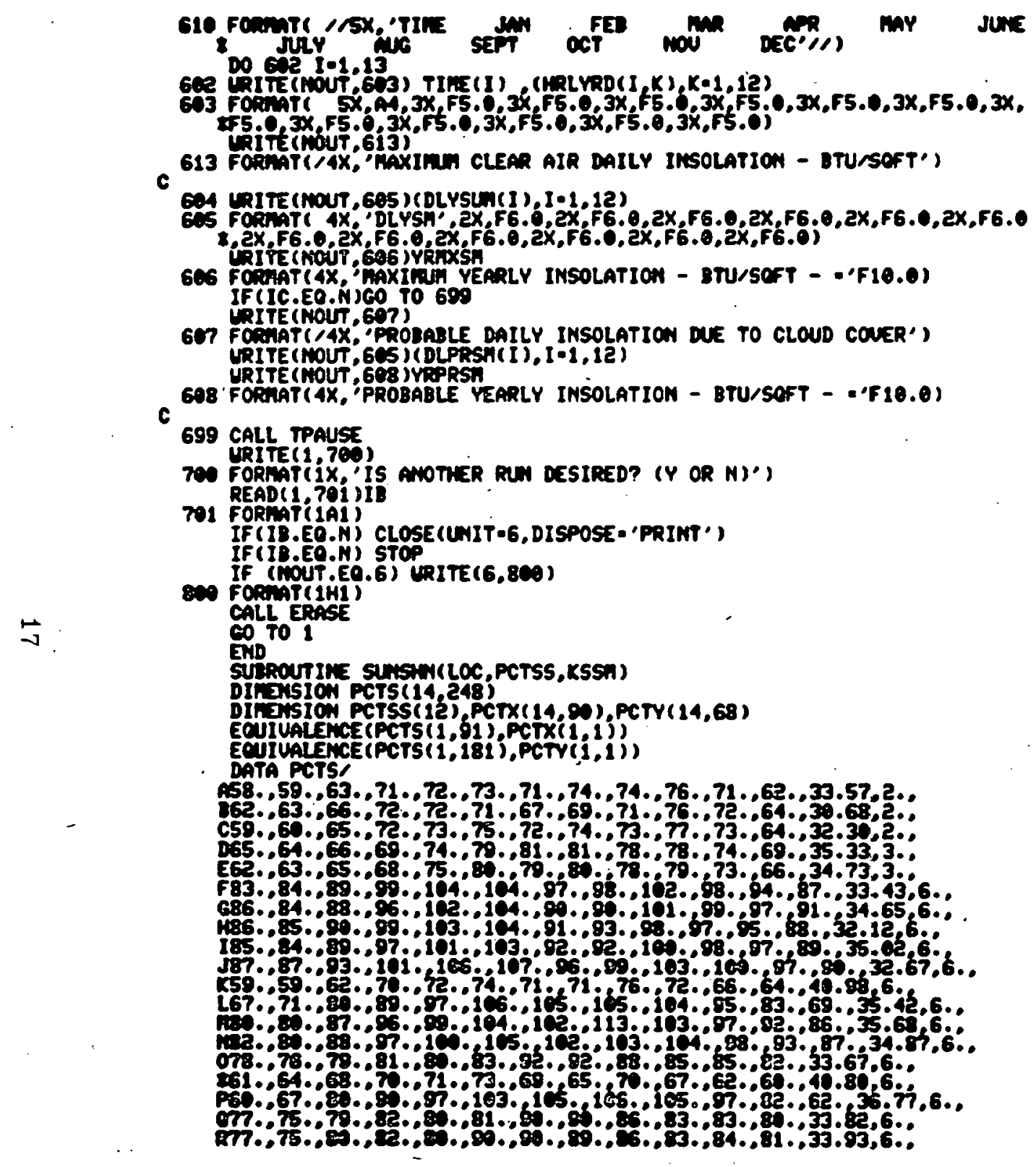




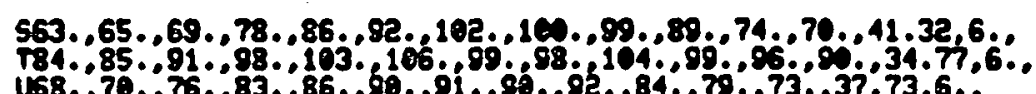

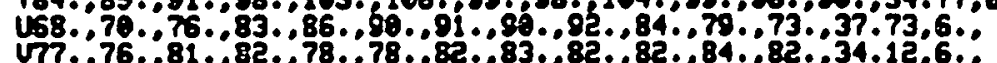
T7.,76. 81.,82.,78.,78.,82.,83,82,82.84, 82.,34.12,6.96

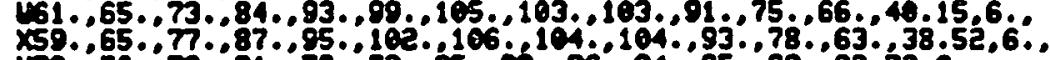
$778 ., 76 ., 79 ., 81 ., 78,, 78 ., 85 ., 83 ., 86 ., 84 ., 85 . .82 ., 32.73,6.0$ $667 ., 69 ., 75 ., 83 ., 87 ., 90 ., 94 ., 93.094 .085 ., 79 ., 72 ., 37.62,6 .$,

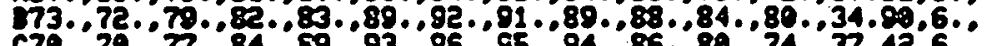
c70..70..7..84.,89..93.,96.,95.,94.886.,80.,74.,37.42,6.,

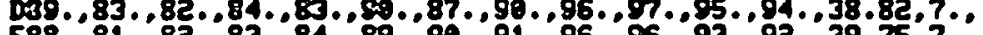

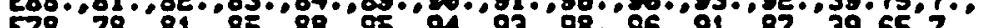
7. (1) 15555 $155 . .57 .0$ K59.

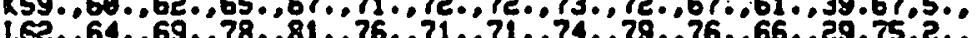

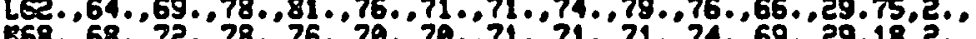
(1)..., (1).,

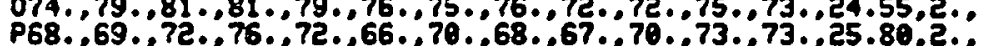

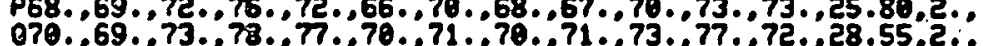

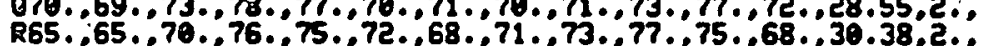

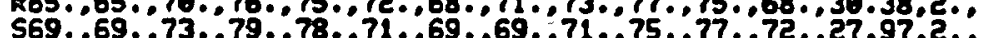

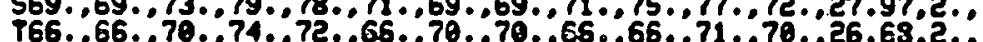

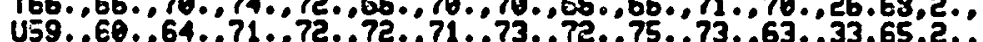

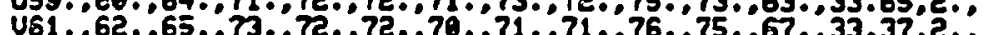
$451.61 .66 .73 .73 .73,70.73 .72,77.7566 .320$

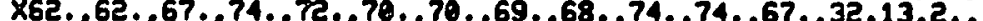

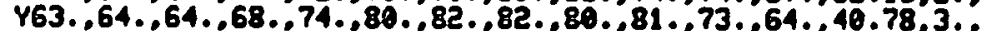
A66. 66. 65. 51 73.89,83,82,82,83,75.68.41,533

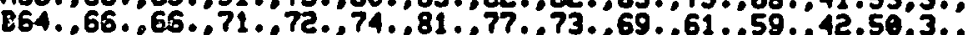
C68.667. D68.

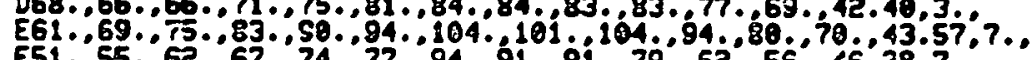

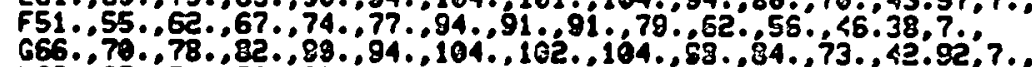

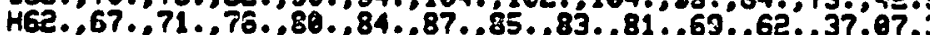
158.,58.,62.,65.,70.76.,77.,78.,78.,76.,65.,57.411.78,3." J60.,62.,62.,65.,69.,75.,77.,77.,78.,77.,68.,60.,41.45,3.: K61..62.,62.,66.,73..80..81.,80.,81..79.,71..62.,39.83,3."

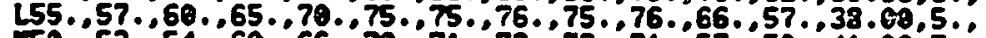
$750 ., 52 ., 54 ., 60 ., 66 ., 70 ., 71 ., 72 ., 73 ., 71 ., 57.550 ., 41.60,5 .$. NS2.,54.,56.,61.,66-.71..71..73.,744.,72.,61.,52.,39.73,5.,

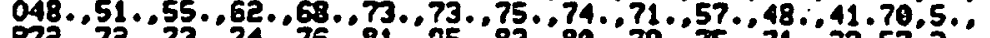
P72..72..73..74.,76.,811..85.,83.,88.,79.,75.771.39.57.3.: a7.

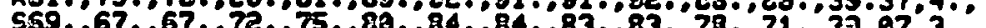
75523.0 153.54 57 . U53.54

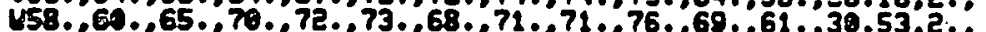

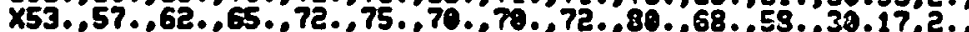
Y61.063.066.,74.,76.,76.,771.72.,74.,77,771.664.,29.98.2. 


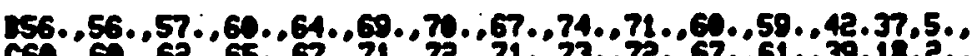

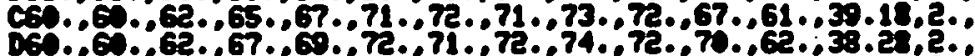

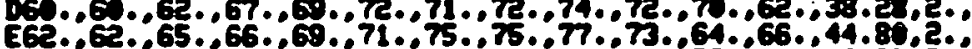

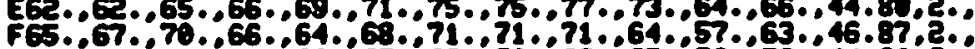

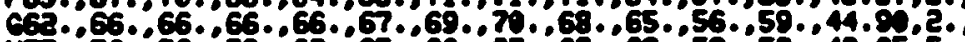

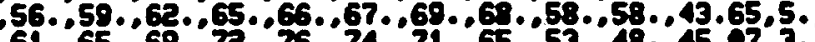
$61.665 ., 69 ., 72 ., 77.09$

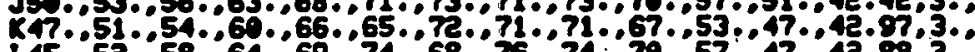

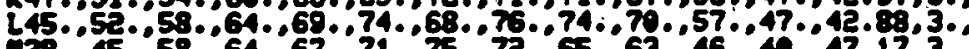

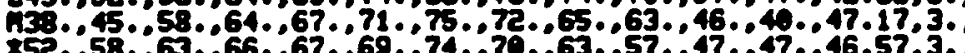
2520

\section{Data PCTX}

M9.,55.,63,.64.,68.,70.,74..72.,67.,62.,50.,50.,46.47,3. 042.48.,51.64.,69.,73.,77.,75.,71.,65.,51.,45.,44.73,3., P60.62.,64.064.,66.68.,75..73.,70.,63.,59.,60.,46.83,3.", .62. ( $561 . .62 .63 .64 .061 ., 43.92,3$. T61..62..62.,66.,73.,79..89..83..80.,79..,71.,63..38.82,3." U65..64.,64.,69.,73.,79.,83.,82.,80.,79..76.,69..39.30,3." 164..62.,64.,69.,73.,78.,81..82.,79.,78.,73.,67.,37.23,3." 163.,62.,64.,68.,73.,78.,81.,89.,80..79..72.,63..38.75,3. X59.,61.,66..72.,75.,77..75..76.,75..77..,71.,63..32.32,2.0 Ys8.,69.,64..70..72.,74..,71..,74.,72.,77..,71.,62.,32.33,2., $160.063 ., 67 . .72 ., 75 ., 71 ., 75 . .77 . .78 . .76 ., 69 ., 57 ., 32.33,2.0$

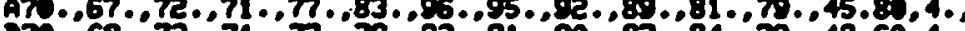
87..68..72..71.,77.,79..93..91.,90..87..84.,79..48.60,4. c73..73..76..75..80..82.096..94.,94..90..84.,81..45.25,4.0 D66..65..70..71..74.,79...9...4.,89.,87..82.,75.,48.22,4.0

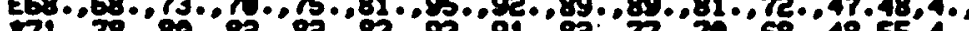

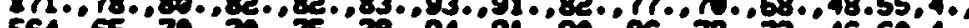
F64..65...7.

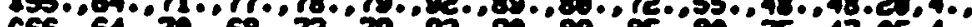
450.0 140.070

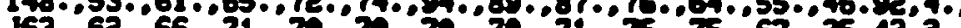
K50.0106.0.0 र

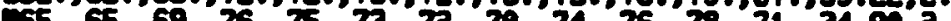

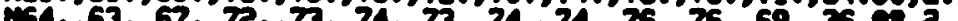
(5) P7. 71.740 24.070 04.6567 .740

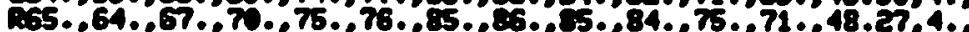

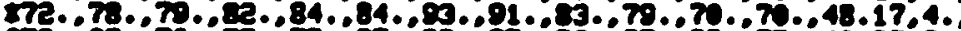
573..69..70..75.,77..85..8..77..4..87..82.,77..4.97.3.0

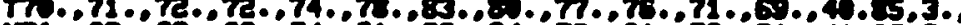

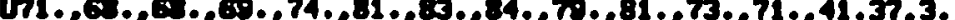

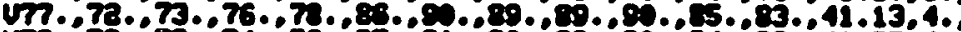

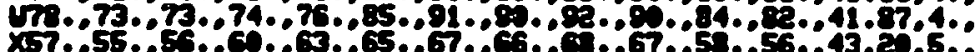

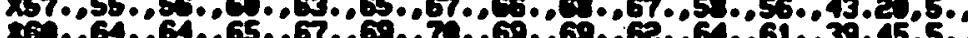

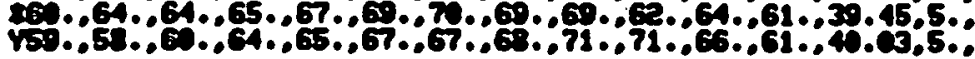




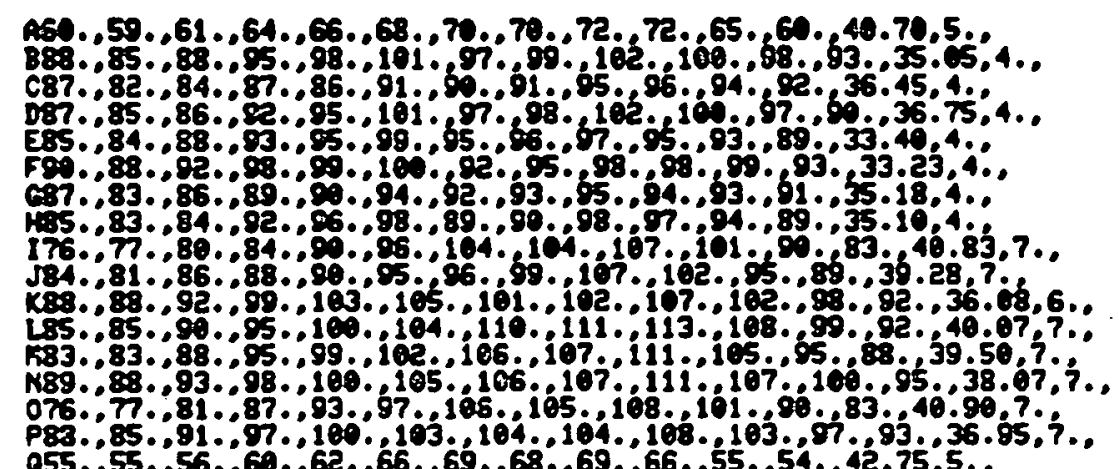

$055 . .55 . .56 . .60 ., 62 ., 66.69 ., 68 ., 69 ., 66 ., 55 ., 54 ., 42.75,5 .$.

R45.,45.,48,.56.,59..64.,66.,65.,66.,62.,49.,43.,42.22,5.,

$543 ., 44 ., 51 ., 59 ., 63 . .69 ., 711.69 ., 68 ., 63 ., 49 ., 43 ., 42.93,5 .$,

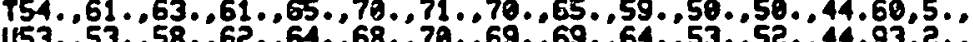

U55.,54..58.,

$460.59 .61 .64 .66 .068 .70 .71 .73 .72 ., 65.61 ., 40,77,5 .$,

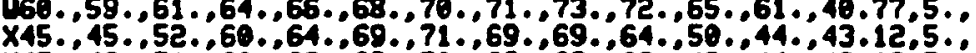

Y47.,46.51.,60.,62.,68.,70.,69.,69.63.,49.,44.,43.12,5.,"

147.,46.,51.,60.,62.,68.,70.,72.,69.,63.,49.,44.,43.12,5.,

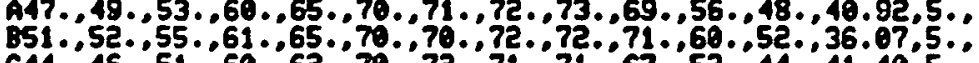

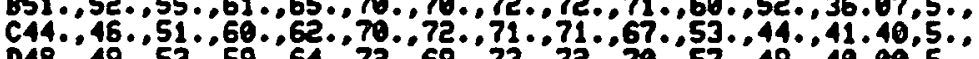

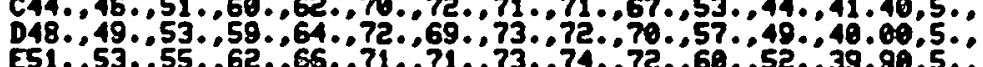

$551 ., 52 ., 55 ., 62 ., 65 ., 71 ., 71 ., 73 ., 74 ., 72 ., 60 ., 52 ., 39.96,5 .$,

$643 ., 44 ., 49 ., 57.62 .67 .69 .68 ., 68.66 .551 .43 .41 .275 .$,

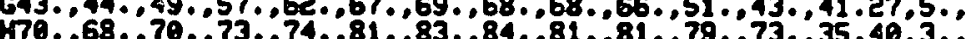

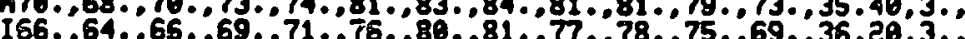

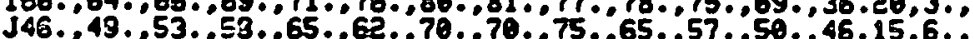

KE2..68.,73..76.,78.,81.,93.,91.,86.,77.,65.'59.,44.83,6."

L62.,65..68.,75..81..87.,98.,95.,97.,87.,75.,69.,43.58,6.:

A48.,58..64.,73..89..87.98.96.993.78.69.50.42.37,6."

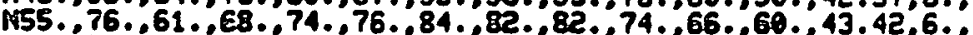

$050.54 ., 63.70 .77 .082 .96 .93 .94081 .63 .55045 .68,6 .$,

P44..49..54.,68.,67.,68.,82.,78.,76.,65.,55.48.45.60,6."

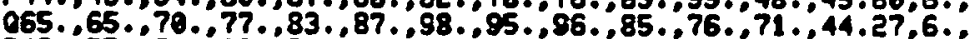

R49.,55.,61..69,.73,.75.,80,.88.,81.,62.,52.,45.,43.22,6.:

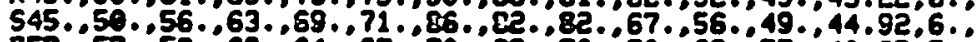

$757 . .57 . .59 ., 62.64, .67 ., 70 . .69 ., 70 ., 70 . .62 ., 57 ., 40.65,5 .$. U40.,45.,52.,61.,65.,70.,73..66.,70.,65.,49.,40.,42.88,5.," DATÁ PCT́Y,

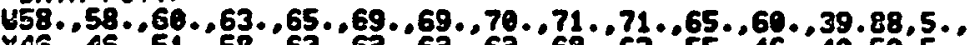
x46..46.,51..58..63.,62.,62..62..68..62..55.,46.,40.58,5.,

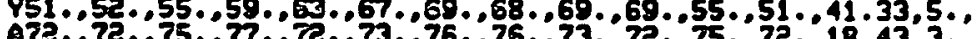

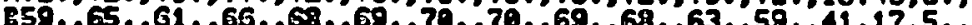

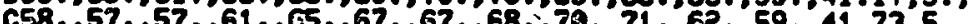

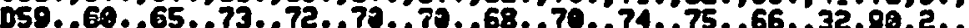

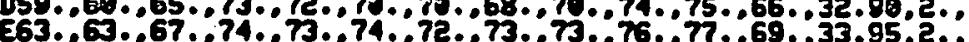
F63..62..66..72..72..73.,72..88.,73.,76.,76.,66.,34.99.2., $665 ., 63 ., 65 ., 70 ., 75 ., 80 ., 87 ., 87 ., 55 ., 84 ., 77 ., 85 ., 44.38,4 .$, 


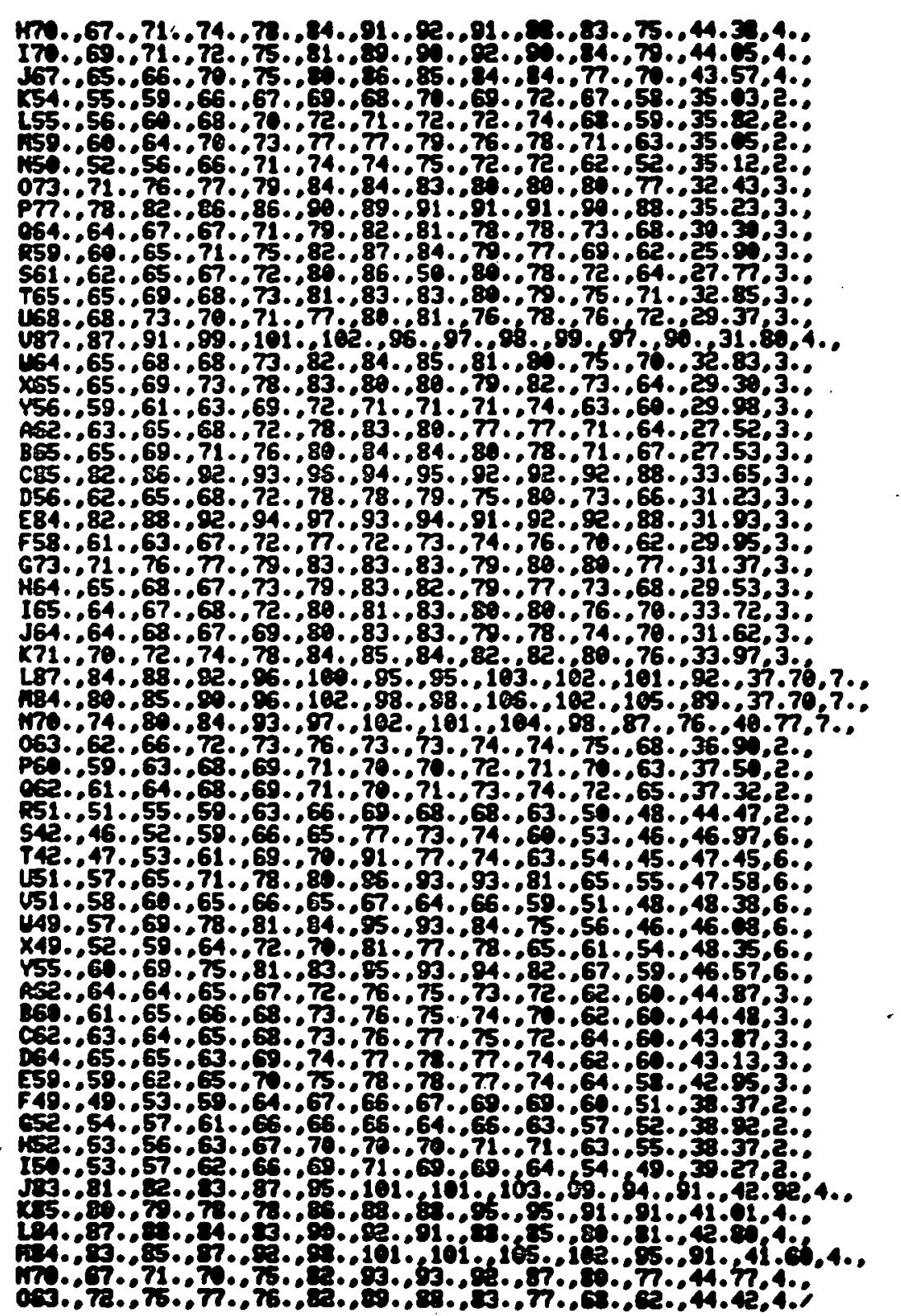


C

sen PCTSS(I)=PCTS(I,LOC)

c

RETUK

PIP) 
ASWRET II

DIhDSSIOW A(12),B(12),C(12), DEC(12), REFLT(12), DLYSUN(12), PAYS(12), PIME (13), BTA(13), SLRAZ (13) KRLYRO(13, 12) DIFRAD 13)

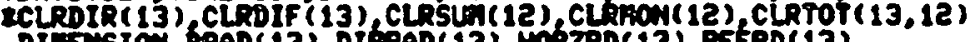

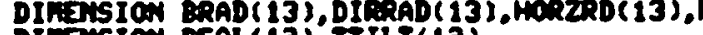

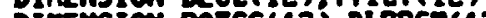

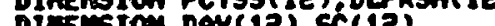

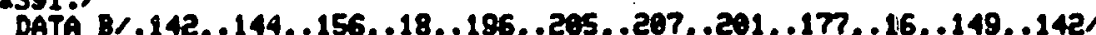
DATA C,.058,.65,.071,.097,.121,.134,.136,.122,.092,.073,.063,.057 DATA DEC $-20 .,-10.8,0 ., 11.6,20 ., 23.45,20.6,12.3,0 .,-10.5,-19.8$, 1-23.45'

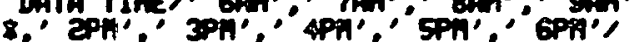

bata pitirio

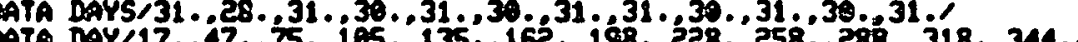

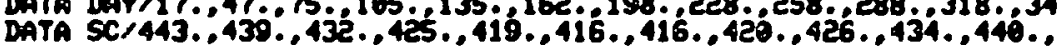

1444.1

DATÁn $N$ N

DTR-57.29578

' I

DOMTU 6 FOR LINE PRIMTER') READ(1, 494), WOUT

c

READ DATA FOR SITE

a VITE(1,2)

FOMATR 'IXNT LATITUE DEG'

ERAD(1,3)LAT

rovintF9.4

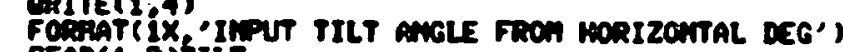
PraD(i, 3 filt

5 FOMAT (iX.'INPUT AZINTM AMELE DES - DUE SOUTH + WEST - EAST') FEAD (2, 3)AZI

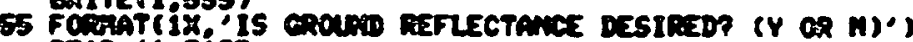
RAD (1.gire IF (IR.EO.N)eo to

MITE(1,6)

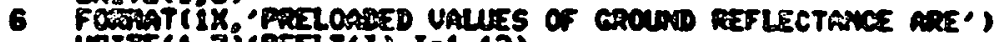

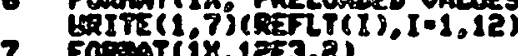

7 Fonvisi, 1 Tr3.8) 
GIIE 11,8$)$

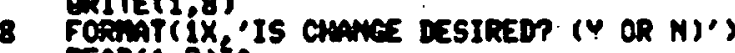

9 rogerif

IF(IA.EQ.H) CO TO 84

19 FOPET(IX 'INPIT 12 ROMTHLY UALLES OF REFLCTANCE')

71 READ $1, ? 1$ i (REFLT $(1), 1-1,12)$

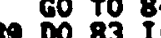

83 DEFIT 1 I 10.12

84 UTt

81 FORmat (1X,'DO YOU DESIRE CLOUD GOUER CALCULATIONS? (Y CR M)' READ (1,9)ÍC IF(IC.EO.H)EO TO 888

LRITE $(1,400)$

400 FORAAT (1X.' IS CITY LOCATION MUABER KNCLN? (Y OR $N$ )', READ(1,9)IL

IF (IL.EO.N) 60 To 80

401 URITE $(1,402)$

402 FOEAAT (IX,'ITPUT CITV LOCATION NHBER'

403 RLAD (1,404)LOC

404 FORAAT(I4)

GO TO 10

92 URITE(1,91) 91 FORMAT (IX,'PRELOADED UALUES OF PERCEMT SUASHIKE RRE') URITE (1,82 ) (FCTSS $(I), I-1,12)$ URITE $(1,8)$ IF (IA. EO

95 URITE(1, 3 ;

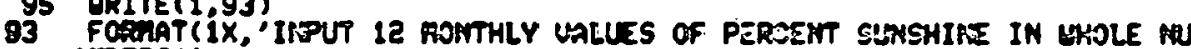
thiEERS' '

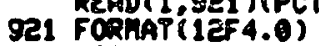

94 KSSHE 1 (1,82)

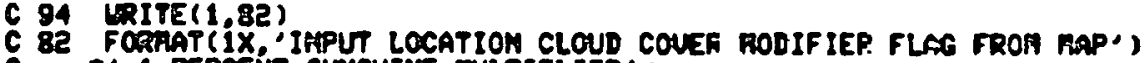

C 1 PERCENT SLOSHIME MULTIPLIER',

I. 3 F

21 4 FRI

8' 5 CLERO AIR THSOLATION')

* 6 F PRIFE TIFES $\left.1.05^{\prime}\right)$

CO 90 i

80800 839

989 CTSS(A)-1\%.

889 contsine

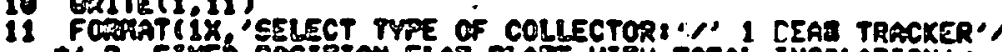

*' 2 FIESO POSIPICN FLAT PLATE UITH TOTAL IlSOLATION"

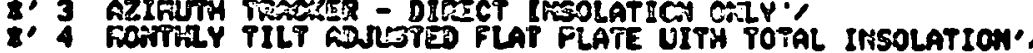




\section{*' 5 FIXED POSITION FLAT PLATE - DIFECT INSOLATION OMY"}

*' 6 MOHHLY TILT ADUSTED FLAT PLATE - DIRECT INSOLATION ONL', TILT TRACKIM CONCEMTRATOR-ENH AXIS ALIGWENT-DIRECT READT I I JKIND

12

c START RTM

Do $221=1,12$

21.

22 CLRSUT(I)=0.

Yerxin=0.

C SET UP TRIG CONSTANTS FOR INPUT MUMBERS

LAT=LAT $/$ DTR

CLAT-COS(LAT)

TLAT=SLAT/CLAT

TIT-TIt八DT

ctit-cos(tilt)

STLT-SIM(TILT)

AZI =AZ1/OTh

SAZ-SIN(AZI)

Dxe-58(1-

DX-.58(1.-CTLT)

Do $1000 n=1,12$

arn

SDECL-SIN(DECL(n))

CDECL-COS(DECL(H))

C TOTAL DAILY HORIZONTAL RADIATION CALC.

HDLnzZ=0.0

cot 1 the $-6,6$

RMGL -15.\& LOAT (ITR) /DTR

Chrugl-cos (KRTGL)

SBTA-CLATECDECLECMRMGL +SLATESDECL

If (SPTA .GT; 1.0)SBTA-1.0

IFCABS(SBTA) -GT. 1.01SBTA=-1.0

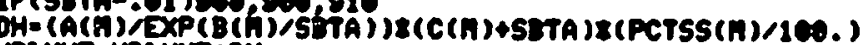

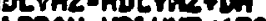

(1)

Triute

c Dally loop for 6 an $10 \mathrm{cpm}$

$\infty$ sen 1 Im:-6,6,1

coprifthto.

Final-15.urionr(1m) 


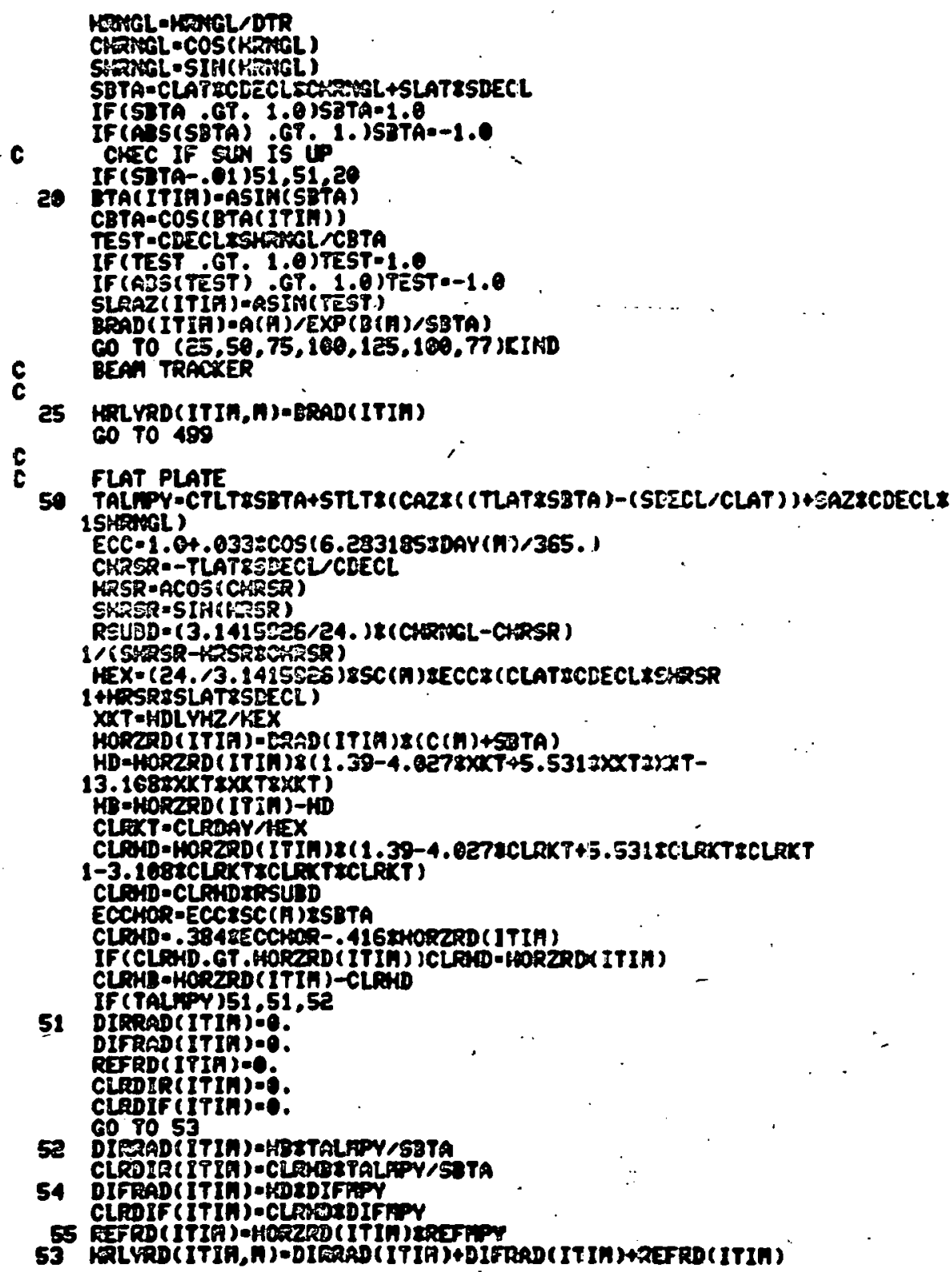




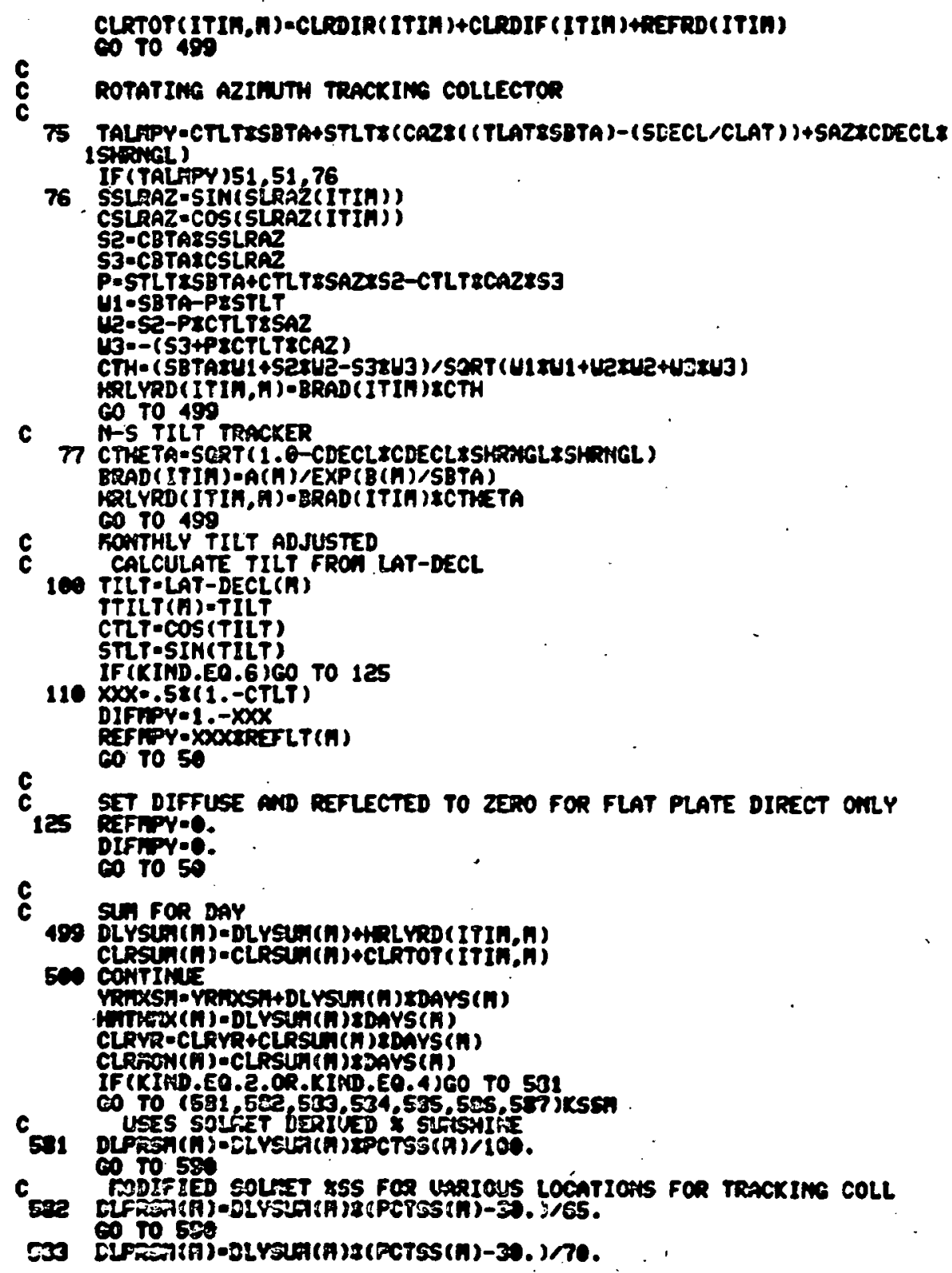




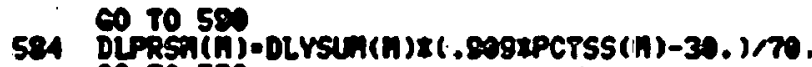

5es Co To $5 \%$

c.5 DLPSN(n)-DLVSTn(n)\&(1.0525tPCTSS (n)-30.1/65.

585 to 50 \%

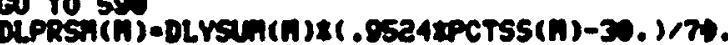

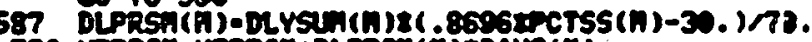

Ien contrut

cat-lateors

TILT-TILTEDTR

AZI =AZIEDTR

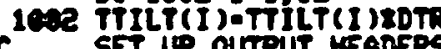

c

60 TO (501,582,503,504,595,506,507)KIND

501 URIFE(MOUT, 511 )

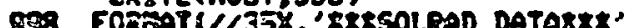

511 FORAATU/23X.' DIRECT IMSOLATIOA FaR BEAN TRACXIME COLLECTORS

*-BTunser - ${ }^{-1}$ '

531 FORMAT(//5X', LATITUDE=' F9.4)

sez Go TO Ges.

512 FGRTAT "/23\%, TOTAL SOLAR INSOLATION FCR FIXED POSITION COLLECTORS

5A2 LRIFE(TFOUT, 552) LAT,TILT AZI

552 FOXPAT ( $15 X$, 'LATITUDE ' F9.4,5X,'TILT=' F9.4,5X,'AZIMTHA ' F9.4)

IF(R.EQ.Nigo To 69

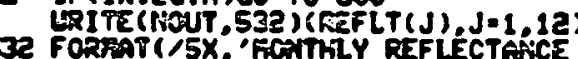

Go 90 6a.

503 URITE(HOUT, 513)

(RITE (FUT' 898 )

513 FORTAT (//88X. 'DIRECT SOLAR INSOLATION FOR ROTATING AZIFUTH TRACKIN 86 COLLECTORS - BTHAHSOFT -'\})

523 URIPE (NOUT, 552 ) LAT, TILT,AZ

6040608

SO4 URITE(NOUT, 514)

514 FORJTAT $/ 13 X^{2}$ 'TOTAL SOLAR INSOLATION FOR MONTHLY TILT ADJUSTED COL

ILECTORS (TILT-LAT-EECL) - BRUH'SOFT -'

524 ERITE(HOUT,534) LAT,AZI

534 Fo.41

SA4 EXITE (NOUT. 554) (TIIT(K), $x-1,12)$

作

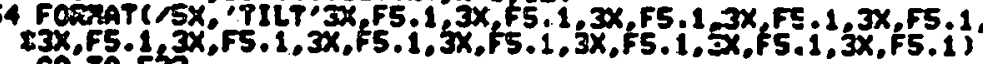

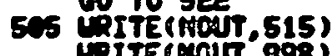

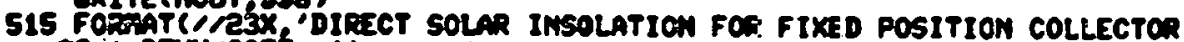
es - Bunserf - 1$$
\text { , }
$$ 
5*6 GITE(MONT,516)

516 FODATU 13 ODIRECT SOLAR IMSOLATION FOR MOWHAY TILT ADJUSTED CO RLECTORS (TIIT-LAT-DECL) - HUHSOFT - ')

CO TO 524

508 FOFATU HIOX 'DIRECT IMSOLATION FOR SOLAR ALTITUDE TRACKING COLLEC ITORS FACING SOUTH ITU/SCF',

URITE(MOUT, 098 )

URITE (NOUT: gTO)LAT

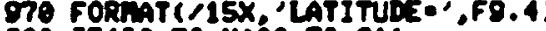

600 IF (IC.EQ.M)GO TO 618

IRITE (ROOUT, G01)LOC

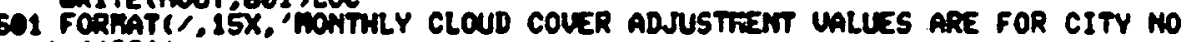

* (13i)

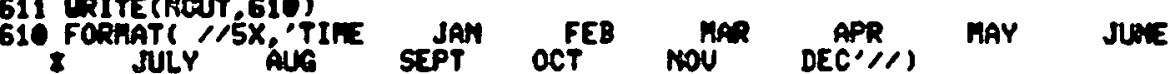
Do $9501=1.13$

IFIKIND.EO.1.OR.KIND.EO.3.OR.KIND.EO.7)GO TO 602 LRITE (MOUT, 603) TIFE (I). (CLRTOT $(I, K), K=1,12$ )

Co 10950

602 LRITE (MOUT, 603) TIKE(I), (HRLYRD(I, K), K-1,12)

950 conimite

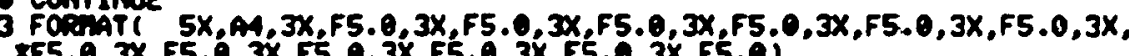
XF5, $0,3 x, 55.6,3 x, F 5.0,3 x, F 5.0,3 x, F 5.0,3 x, F 5.01$

613 FOOAT (4X, ' AXImO CLEAR AIR DAILY IMSOLATIOY - BTURSOFT') IFIKIND.EO'1.OR.KIMD.EO.3.OR.KIND.EO.7ICO TO 604 Do 951 i=1.12

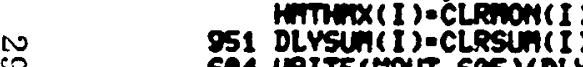

951 DLYSUn(1)-CLrsun( (1)

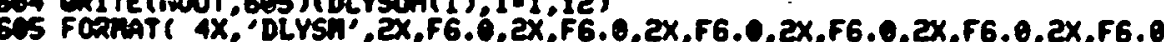

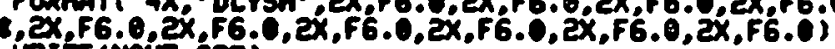

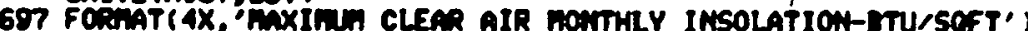

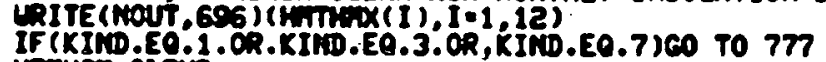
Vorxsin-CLOY

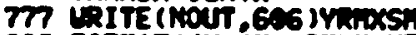

6 FOORAT(4X, MAXIMUH YEARLY INSOLATION - BTU/SOFT - -'F10.0) IF (IC.KE.N)GO TO 608

695 Foof $1 F 6.0,2 x, F 6.0,2 x, F 6.0,2 x, F 6.0,2 x, F 6.0,2 x, F 6.0)$

co ro 609 .

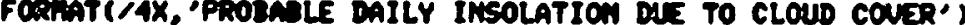

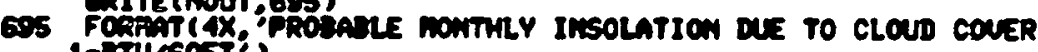
1-Tursert', ATE (Foyr.698) (1)

$c$

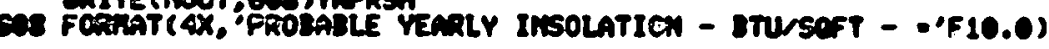

cos calt Trauser, 


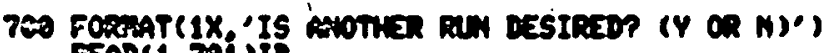

TEAD(1,701 II.

IF(IT.EO.N) CLOSE(UNIT-6,DISPOSE- PRIHT')

IFi I. E0.Ni sto

890 calt ERase

Go 701

END.

SUPOUTIE: SLUSH(LOC,PCTSS,KSST)

Difretsion PCTSS(12),PCTX(14,9),PCTY $(14,68)$

EGUTUALENCE(PCTS $(1,91), P C T X(1,1))$

DATA PCTS'
$658 ., 59 ., 63 ., 71 . .72 . .73 ., 71 . .74 . .74 ., 76 . .71 ., 62 ., 33.57 .2 .$.

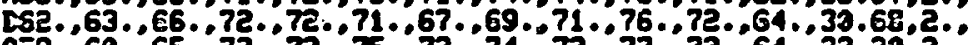

C59.,60..65..72..73..75.,72.,74..73.,77.,73..64.,32.39,2..

L55..64..66..69..74.,79.,81.,81.78.,78.,74.,69.,35.33,3."

F83.84.89.98.

C86.84.88.86. 102.104.90.98.9101,99.97.91.34.65.6.

Hes. 45.58 .09 .03 .104 .91 .93 .98 .97 .95 .83 .32 .126 .0

$185.84 .89 .97,161.103 .92 .02,168.98 .97 .89 .35 .026$

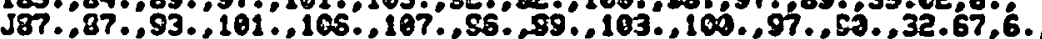

$559.059 .62 .70 .72 .74,71,71,76.72 .65 .64 ., 40.986$.

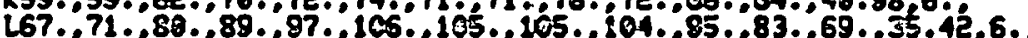

R59.

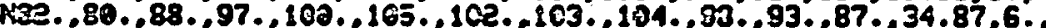

78.,76.,79.,81..82.,83.,82..,82.,83..85.,85..82..33.67.6..

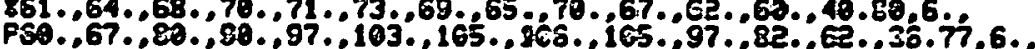

$077 ., 75 ., 79 ., 82 ., 60 ., 81 ., 50 . .83 ., C 5 ., 83 ., 63 ., 80 ., 33.22,6$.

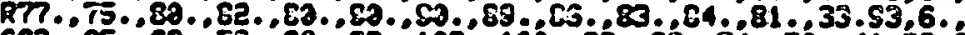

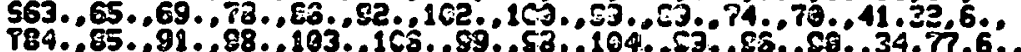

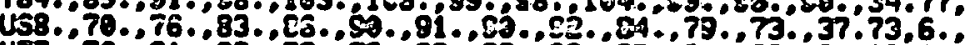

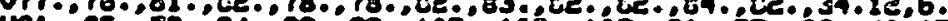

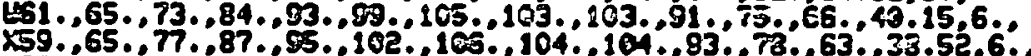

$78.76 .79 .81 .78 ., 73.85 ., 63.06 .84 . .65 ., 02 ., 32.73,6 \ldots$

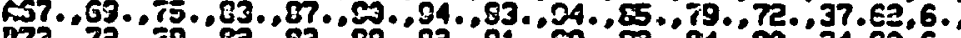

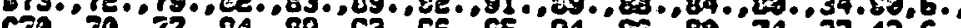

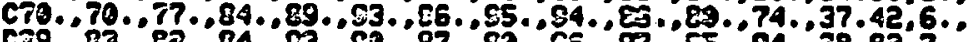

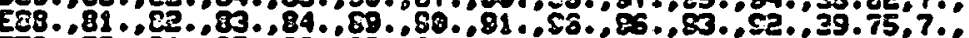

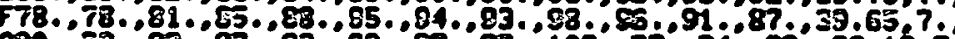

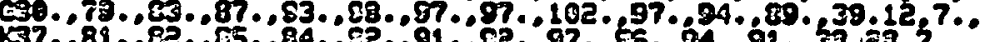

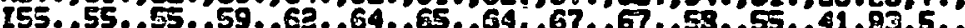

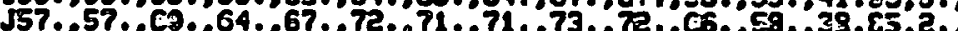

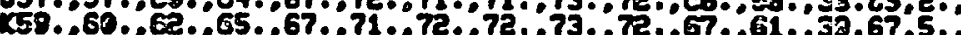

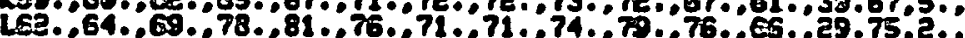

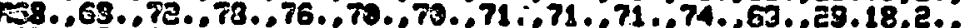

.07.067.. .

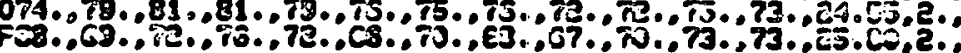




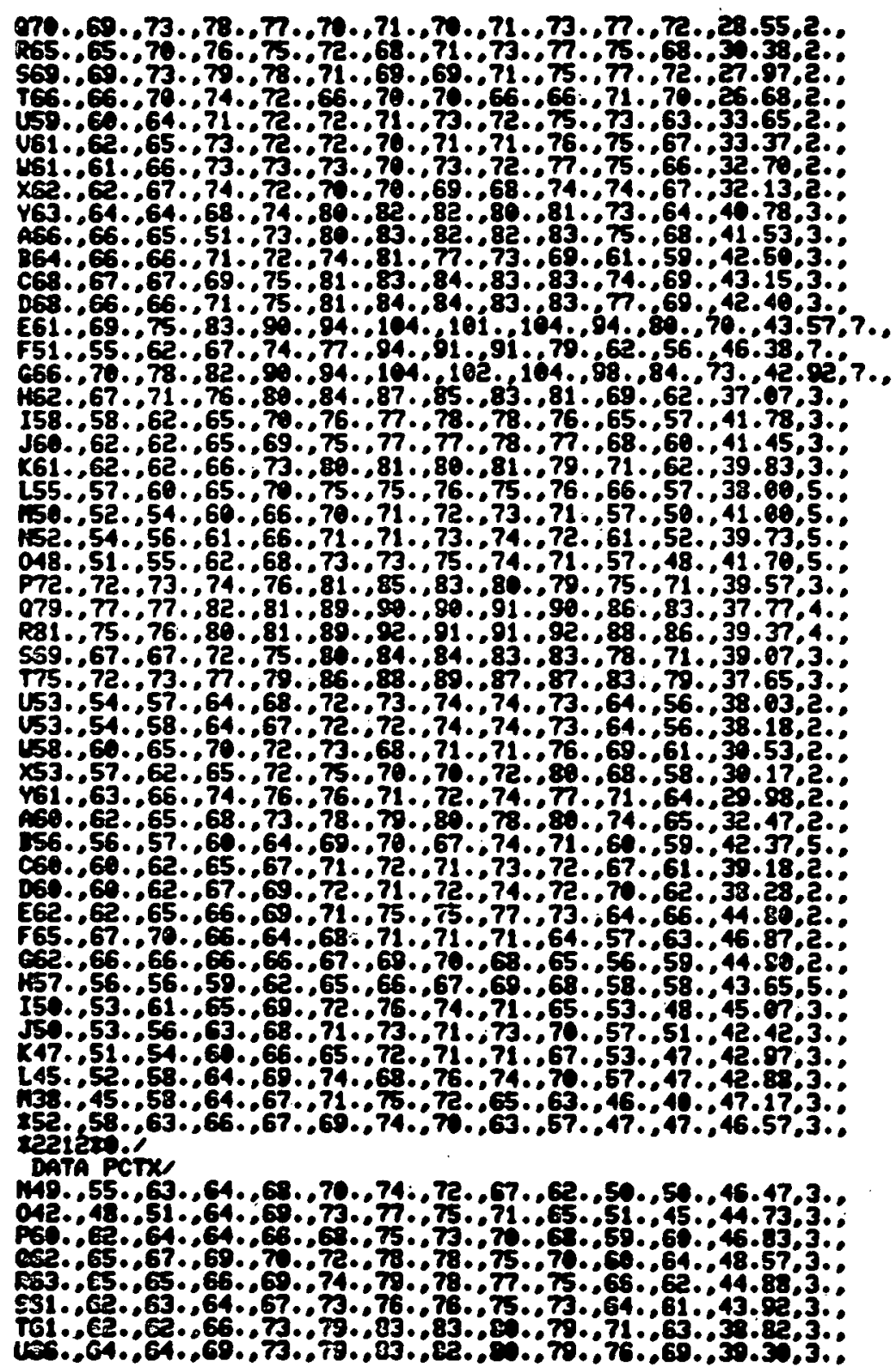




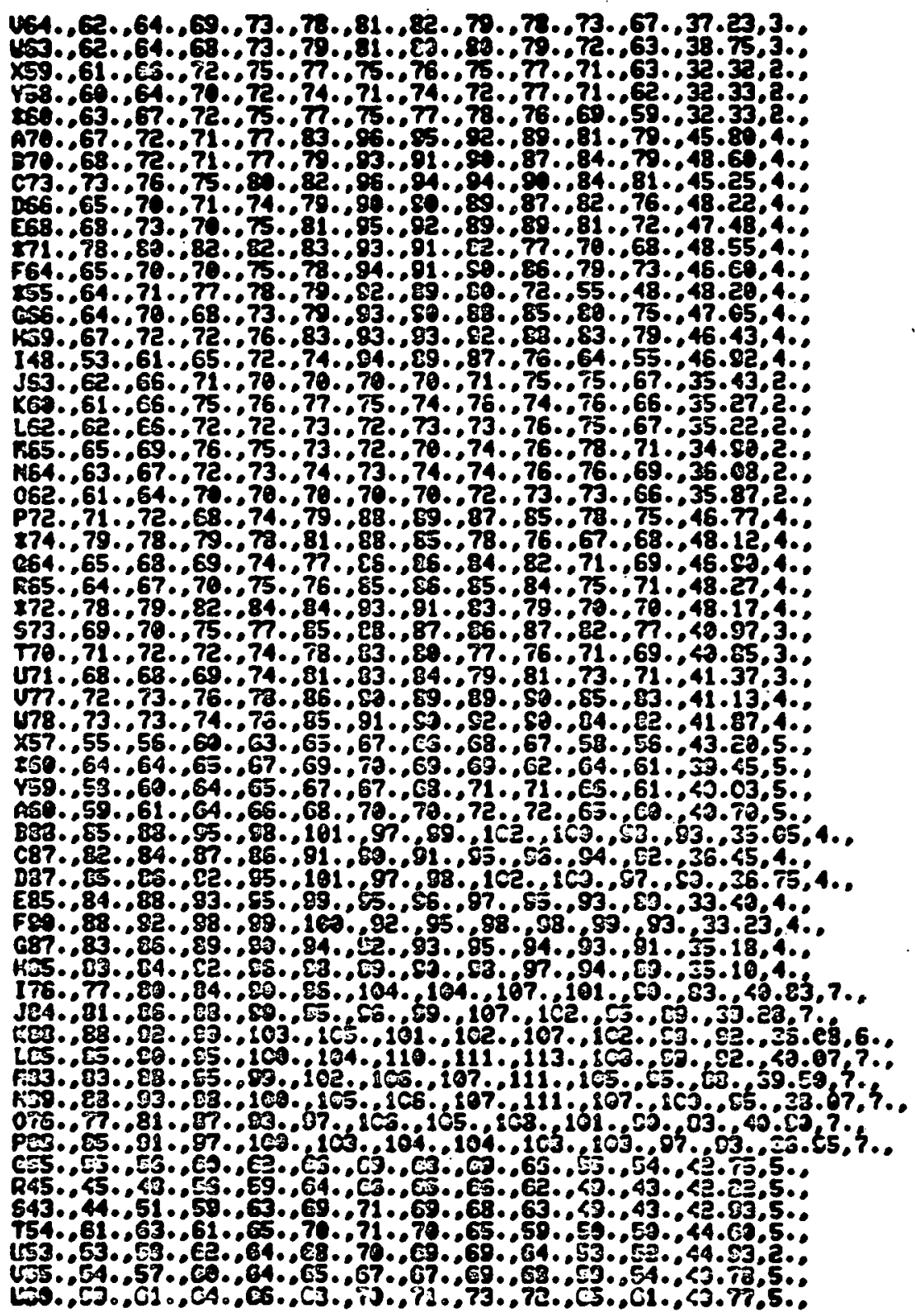




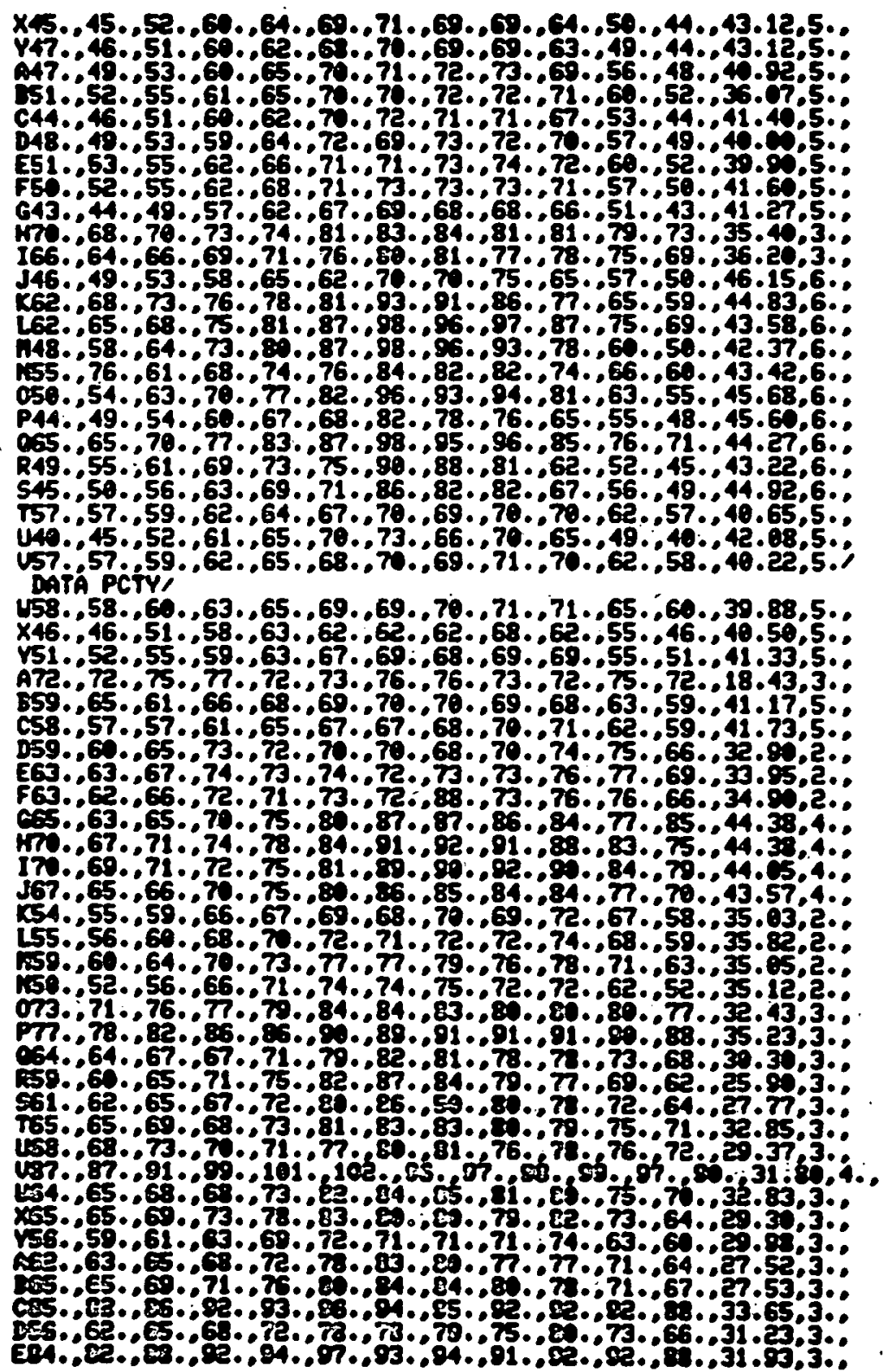




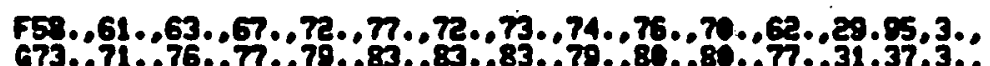

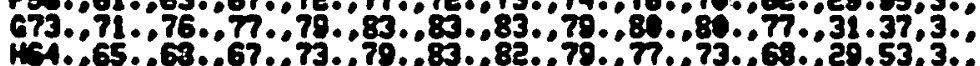

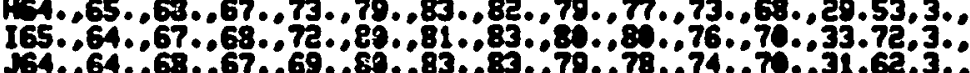

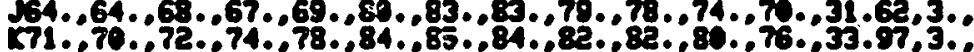

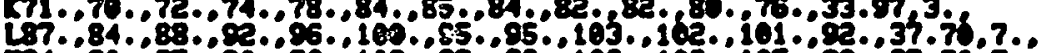

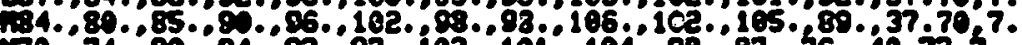

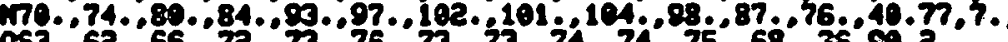

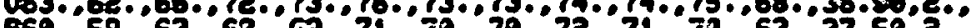
(15. (5) 54.56

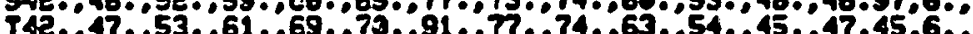

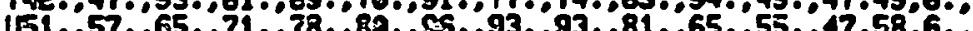
55 4099. $\times 49.52 .59040 .78$

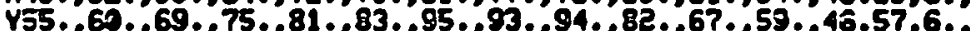

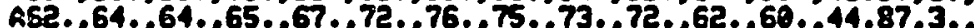
$860.61 .65 .66568 .73,76.75 .74 .70 .62 .69 .44 .483$

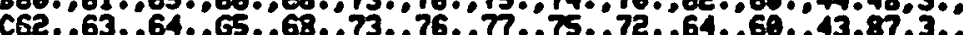
664.65 .65 .63 .69 .074 .77 .78 .77 .74 .62 .69 .43 .13 .3

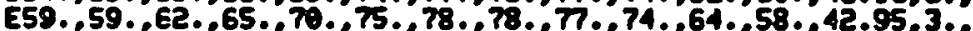

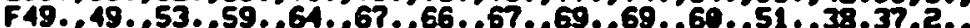
652.056 .920 年, $150 . .53 ., 57 ., 62 ., 66 ., 69 ., 71,69 ., 69,64,54,049 ., 39.27,2.0$ J33.,81.,82.,83..87..55.,101..101,103.,99,.94,911,42.92,4., K85..80.,79..78.,78.,856.,88.,88..55.,95..91.,91..41.91.4..

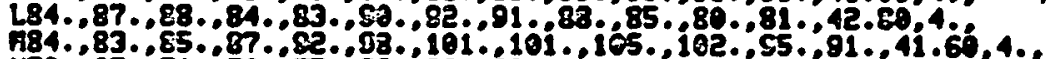

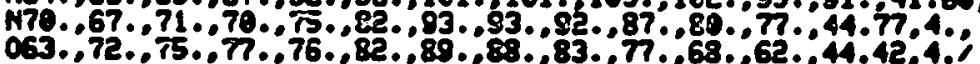

500 $5001=1.12$

500 PCTSS(I)=PCTS(I,LOC)

c extuen

PIP) 
TABLE 1. ASHMET PROGRAM CITY LIST

\begin{tabular}{|c|c|c|c|c|c|c|c|c|}
\hline No. & STATION AND STATE & LAT. $\sim^{\circ}$ & No. & STATION AND STATE & LAT. $\sim^{0}$ & No. & STATION AND STATE & LAT. $\sim^{\circ}$ \\
\hline 1 & BIRMINGHAM, AL & 33.57 & 46 & W. PALM BEACH, FL & 26.68 & 91 & SAULT STE. MARIE, MI & 46.47 \\
\hline 2 & MOBILE, AL & 30.68 & 47 & ATLANTA, GA & 33.65 & 92 & TRAVERSE CITY, MI & 44.73 \\
\hline 3 & MONTGOMERY,AL & 32.30 & 48 & AUGUSTA, GA & 33.37 & 93 & DULUTH, MN & 46.83 \\
\hline 4 & FT. SMITH, AR & 35.33 & 49 & MACON, GA & 32.70 & 94 & INTERNATIONAL FALLS, MN & 48.57 \\
\hline $\mathbf{5}$ & LITTLE ROCK, AR & 34.73 & 50 & SAVANNAH, GA & 32.13 & 95 & MINNEAPOLIS-ST.PAUL, MN & 44.88 \\
\hline 6 & PHOENIX, AZ & $\mathbf{3 3 . 4 3}$ & 51 & BURLINGTON, IA & 40.78 & 96 & ROCHESTER, MN & 43.92 \\
\hline 7 & PRESCOTT, AZ & 34.65 & 52 & DES MOINES, IA & 41.53 & 97 & COLUMBIA, MO & 38.82 \\
\hline 8 & TUCSON, AZ & 32.12 & 53 & DUBUQUE, IA & 42.50 & 98 & KANSAS CITY, MO & 39.30 \\
\hline 9 & WINSLOW, AZ & 35.02 & 54 & MASON CITY, IA & 43.15 & 99 & SPRINGFIELD, MO & 37.23 \\
\hline 10 & YUMA, AZ & 32.67 & 55 & SOUIX, CITY, IA & $\mathbf{4 2 . 4 0}$ & 100 & ST. LOUIS, MO & 38.75 \\
\hline 11 & ARCATA, CA & 40.98 & 56 & BOISE, ID & 43.57 & 101 & JACKSON, MS & 32.32 \\
\hline 12 & BAKERSFIELD, CA & $\mathbf{3 5 . 4 2}$ & 57 & LEWISTON, ID & 46.38 & 102 & MERIDIAN, MS & 32.33 \\
\hline 13 & CHINA LAKE, CA & 35.68 & 58 & POCATELLO, ID & 42.92 & 103 & VICKSBURG, MS & 32.33 \\
\hline 14 & DAGGETT, CA & 34.87 & 59 & CAIRO, IL & 37.07 & 104 & BILLINGS, MT & 45.80 \\
\hline 15 & EL TORO, CA & 33.67 & 60 & CHICAGO, IL & 41.78 & 105 & CUT BANK, MT & 48.60 \\
\hline 16 & EUREKA, CA & 40.80 & 61 & MOLINE, IL & 41.45 & 106 & DILLON, MT & 45.25 \\
\hline 17 & FRESNO, CA & 36.77 & 62 & SPRINGFIELD, IL & 39.83 & 107 & GLASGOW, MT & 48.22 \\
\hline 18 & LONG BEACH, CA & 33.82 & 63 & EVANSVILLE, IN & $\mathbf{3 8 . 0 0}$ & 108 & GREAT FALLS, MT & 47.48 . \\
\hline 19 & LOS ANGELES, CA & 33.93 & 64 & FORT WAYNE, IN & 41.00 & 109 & HAVRE, MT & 48.55 \\
\hline 20 & MT. SHASTA, CA & 41.32 & 65 & INDIANAPOLIS, IN & 39.73 & 110 & HELENA, MT & 46.60 \\
\hline 21 & NEEDLES. CA & 34.77 & 66 & SOUTH BEND, IN & 41.70 & 111 & KALISPELL, MT & 48.20 \\
\hline 22 & OAKLAND, CA & 37.73 & 67 & CONCORDIA, KS & 39.57 & 112 & LEWISTON, MT & 47.05 \\
\hline 23 & PT. MUGU, CA & 34.12 & 68 & DODGE CITY, KS & 37.77 & 113 & MILES CITY, MT & 46.43 \\
\hline 24 & RED BLUFF, CA & 40.15 & 69 & GOODLAND. KS & 39.37 & 114 & MISSOULA, MT & 46.92 \\
\hline 25 & SACRAMENTO, CA & 38.52 & 70 & TOPEKA, KS & 39.07 & 115 & ASHVILLE, NC & 35.43 \\
\hline 26 & SAN DIEGO, CA & 32.73 & 71 & WICHITA, KS & 37.65 & 116 & CAPE HATTERAS, NC & 35.27 \\
\hline 27 & SAN FRANCISCO, CA & 37.62 & 72 & LEXINGTON, KY & 38.03 & 117 & CHARLOTTE, NC & 35.22 \\
\hline 28 & SANTA MARIA, CA & 34.90 & 73 & LOUISVILLE, KY & 38.18 & 118 & CHERRY POINT, JNC & 34.90 \\
\hline 29 & SUNNYVALE, CA & 37.42 & 74 & BATON ROUGE, LA & 30.53 & 119 & GREENSBORO, NC & 36.08 \\
\hline 30 & COLORADO SPRINGS, CO & 38.82 & 75 & LAKE CHARLES, LA & 30.17 & 120 & RALEIGH-DURHAM, NC & 35.87 \\
\hline 31 & DENVER, CO & 39.75 & 76 & NEW ORLEANS, LA & 29.98 & 121 & BISMARK, ND & 46.77 \\
\hline 32 & EAGLE, CO & 39.65 & 77 & SHREVEPORT. LA & 32.47 & 122 & DEVILS LAKE, ND & 48.12 \\
\hline 33 & GRAND JUNCTION. CO & 39.12 & 78 & BOSTON, M.A & 42.37 & 123 & FARGO, ND & 46.90 \\
\hline 34 & PUEBLO, CO & 38.28 & 79 & BALTIMORE, MD & 39.18 & 124 & MINOT; ND & 48.27 \\
\hline 35 & HARTFORD, CT & 41.93 & 80 & PATUXENT RIVER, MD & 38.28 & 125 & WILLISTON, ND & 48.17 \\
\hline 36 & WASHINGTON, DC & $\mathbf{3 8 . 8 5}$ & 81 & BANGOR, ME & 44.80 & 126 & GRAND ISLAND, NE & 40.97 \\
\hline 37 & WILMINGTON, DE & 39.67 & 82 & CARIBOU, ME & 46.87 & 127 & LINCOLN, NE & $\mathbf{4 0 . 8 5}$ \\
\hline 38 & APALACHICOLA, FL & 29.75 & 83 & EASTPORT, ME & 44.90 & 128 & NORTH OMAHA, NE & 41.37 \\
\hline 39 & DAYTONA BEACH, FL & 29.18 & 84 & PORTLAND. ME & 43.65 & 129 & NORTH PLATT, NE & 41.13 \\
\hline 40 & JACKSONVILLE, FL & 30.50 & 85 & ALPENA, MI & 45.07 & 130 & SCOTTSBLUFF, NE & 41.87 \\
\hline 41 & KEY WEST, FL & 24.55 & 86 & DETROIT, NII & 42.42 & 131 & CONCORD, NH & 43.20 \\
\hline 42 & MIAMI, FL & 25.80 & 87 & FLINT, MI & 42.97 & 132 & ATLANTIC CITY, NJ & 39.45 \\
\hline 43 & ORLANDO, FL & 28.55 & 88 & GRAND RAPIDS, MI & 42.88 & 133 & LAKEHURST, NJ & 40.03 \\
\hline 44 & TALLAHASSEE, FL & 30.38 & 89 & HOUGHTON, M! & 47.17 & 134 & NEWARK, NJ & 40.70 \\
\hline 45 & TAMPA, FL & 27.97 & $\mathbf{9 0}$ & MARQUETTE, MI & 46.57 & 135 & ALBUQUEROUE, NM & 35.05 \\
\hline
\end{tabular}


TABLE 1. CONCLUDED

\begin{tabular}{|c|c|c|c|c|c|c|c|c|}
\hline No. & STATION AND STATE & LAT. $\sim^{\circ}$ & No. & STATION AND STATE & LAT. $\sim^{\circ}$ & No. & STATION AND STATE & LAT. ${ }^{\circ}$ \\
\hline 136 & CLAYTON, NM & 36.45 & 180 & HARRISBURG, PA & 40.22 & 223 & NORFOLK, VA & 36.90 \\
\hline 137 & FARMINGTON, NM & 36.75 & 181 & PHILADELPHIA, PA & 39.88 & 224 & ZICHMOND, VA & 37.50 \\
\hline 138 & ROSWELL, NM & $\mathbf{3 3 . 4 0}$ & 182 & PITTSBURG, PA & 40.50 & 225 & ZOANOKE, VA & 37.32 \\
\hline 139 & TRUTH OR CONSEQUENCES, & 33.23 & 183 & WILKES-BARRE- & & 226 & BURLINGTON, VT & 44.47 \\
\hline & & & & SCRANTON, PA & 41.33 & 227 & JLYMPIA, WA & 46.97 \\
\hline 140 & TUCUMCARI, NM & 35.18 & 184 & SAN JUAN, PR & 18.43 & 228 & SEATTLE-TACOMA, WA & 47.45 \\
\hline 141 & ZUNI, NM & 35.10 & 185 & BLOCK ISLAND, RI & 41.17 & 229 & SPOKANE, WA & 47.58 \\
\hline 142 & ELKO, NV & 40.83 & 186 & PROVIDENCE, RI & 41.73 & 230 & TATOOSH ISLAND, WA & 48.38 \\
\hline 143 & ELY, NV & 39.28 & 187 & CHARLESTON, SC & 32.90 & 231 & WALLA WALLA, WA & 46.08 \\
\hline 144 & LAS VEGAS, NV & 36.08 & 188 & COLUMBIA, SC & 33.95 & 232 & WHIDBEY ISLAND, WA & 48.35 \\
\hline 145 & LOVELOCK, NV & 40.07 & 189 & GREENVILLE- & & 233 & YAKIMA, WA & 46.57 \\
\hline 146 & RENO, NV & 39.50 & & SPARTANBURG, SC & 34.90 & 234 & EAU CLAIRE, WI & 44.87 \\
\hline 147 & TONOPAH, NV & 38.07 & 190 & HURON, SD & 44.38 & 235 & GREENBAY, WI & 44.48 \\
\hline 148 & WINNEMUCCA, NV & 40.90 & 191 & PIERRE, SD & 44.38 & 236 & LA CROSSE, WI & 43.87 \\
\hline 149 & YUCCA FLATS, NV & 36.95 & 192 & RAPID CITY, SD & $\mathbf{4 4 . 0 5}$ & 237 & MADISON, WI & 43.13 \\
\hline 150 & ALBANY, NY & 42.75 & 193 & SỌUIX FALLS, SD & 43.57 & 238 & MILWAUKEE, WI & 42.95 \\
\hline 151 & BINGHAMTON, NY & 42.22 & 194 & CHATTANOOGA, TN & 35.03 & 239 & CHARLESTON, WV & 38.37 \\
\hline 152 & BUFFALO, NY & 42.93 & 195 & KNOXVILLE, TN & 35.32 & 240 & ELKINS, WV & 38.92 \\
\hline 153 & CANTON, NY & 44.60 & 196 & MEMPHIS, TN & 35.05 & 241 & HUNTINGTON, WV & 38.37 \\
\hline 154 & MASSENA, NY & 44.93 & 197 & NASHVILLE, TN & 35.12 & 242 & PARKERSBURG, WV & 39.27 \\
\hline 155 & NYC (CENTRAL PARK), NY & 40.78 & 198 & ABILENE, TX & 32.43 & 243 & CASPER, WY & 42.92 \\
\hline 156 & NYC (LA GUARDIA), NY & 40.77 & 199 & AMARILLO, TX & 35.23 & 244 & CHEYENNE, WY & 41.01 \\
\hline 157 & ROCHESTER, NY & 43.12 & 200 & AUSTIN, TX & 30.30 & 245 & LANDER, WY & 42.80 \\
\hline 158 & SYRACUSE, NY & 43.12. & 201 & BROWNSVILLE, TX & 25.90 & 246 & ROCK SPRINGS, WY & 41.60 \\
\hline 159 & AKRON-CANTON, OH & 40.92 & 202 & CORPUS CHRISTI, TX & 27.77 & 247 & SHERIDAN, WY & 44.77 \\
\hline 160 & CINCINNATI, OH & 36.07 & 203 & DALLAS, TX & 32.85 & 248 & YELLOWSTONE PARK, WY & 44.42 \\
\hline 161 & CLEVELAND, OH & 41.40 & 204 & DEL RIO, TX & 29.37 & & & \\
\hline 162 & COLUMBUS, OH & $\mathbf{4 0 . 0 0}$ & 205 & EL PASO, TX & 31.80 & & & \\
\hline 163 & DAYTON, OH & 39.90 & 206 & FORT WORTH, TX & 32.83 & & & \\
\hline 164 & TOLEDO, OH & 41.60 & 207 & GALVESTON, TX & 29.30 & & & \\
\hline 165 & YOUNGSTOWN, OH & 41.2? & 208 & HOUSTON, TX & 29.98 & & & \\
\hline 166 & OKLAHOMA CITY, OK & $\mathbf{3 5 . 4 0}$ & 209 & KINGSVILLE, TX & 27.52 & & & \\
\hline 167 & TULSA, OK & 36.20 & 210 & LAREDO, TX & 27.53 & & & \\
\hline 168 & ASTORIA, OR & 46.15 & 211 & LUBBOCK, TX & 33.65 & & & \\
\hline 169 & BAKER, OR & $\mathbf{4 4 . 8 3}$ & 212 & LUFKIN, TX & 31.23 & & . & \\
\hline 170 & BURNS, OR & 43.58 & 213 & MIDLAND-ODESSA, TX & 31.93 & & & \\
\hline 171 & MEDFORD, OR & 42.37 & 214 & PORT AUTHUR, TX & 29.95 & & & \\
\hline 172 & NORTH BEND, OR & 43.42 & 215 & SAN ANGELO, TX & 31.37 & & & \\
\hline 173 & PENDLETON, OR & 45.68 & 216 & SAN ANTONIA, TX & 29.53 & & & \\
\hline 174 & PORTLAND, OR & 45.60 & 217 & SHERMAN, TX & 33.72 & & & \\
\hline 175 & REDMOND, OR & 44.27 & 218 & WACO, TX & 31.62 & & r & \\
\hline 176 & ROSEBURG, OR & 43.22 & 219 & WICHITA FALLS, TX & $33: 97$ & & & \\
\hline 177 & SALEM, OR & 49.44 & 220 & BRYCE CANYON, UT & $\mathbf{3 7 . 7 0}$ & & & \\
\hline 178 & ALLENTOWN, PA & 40.65 & 221 & CEDAR CITY, UT & $\mathbf{3 7 . 7 0}$ & & & \\
\hline 179 & ERIE, PA & 42.08 & 222 & SALT LAKE CITY, UT & 40.77 & & & \\
\hline
\end{tabular}




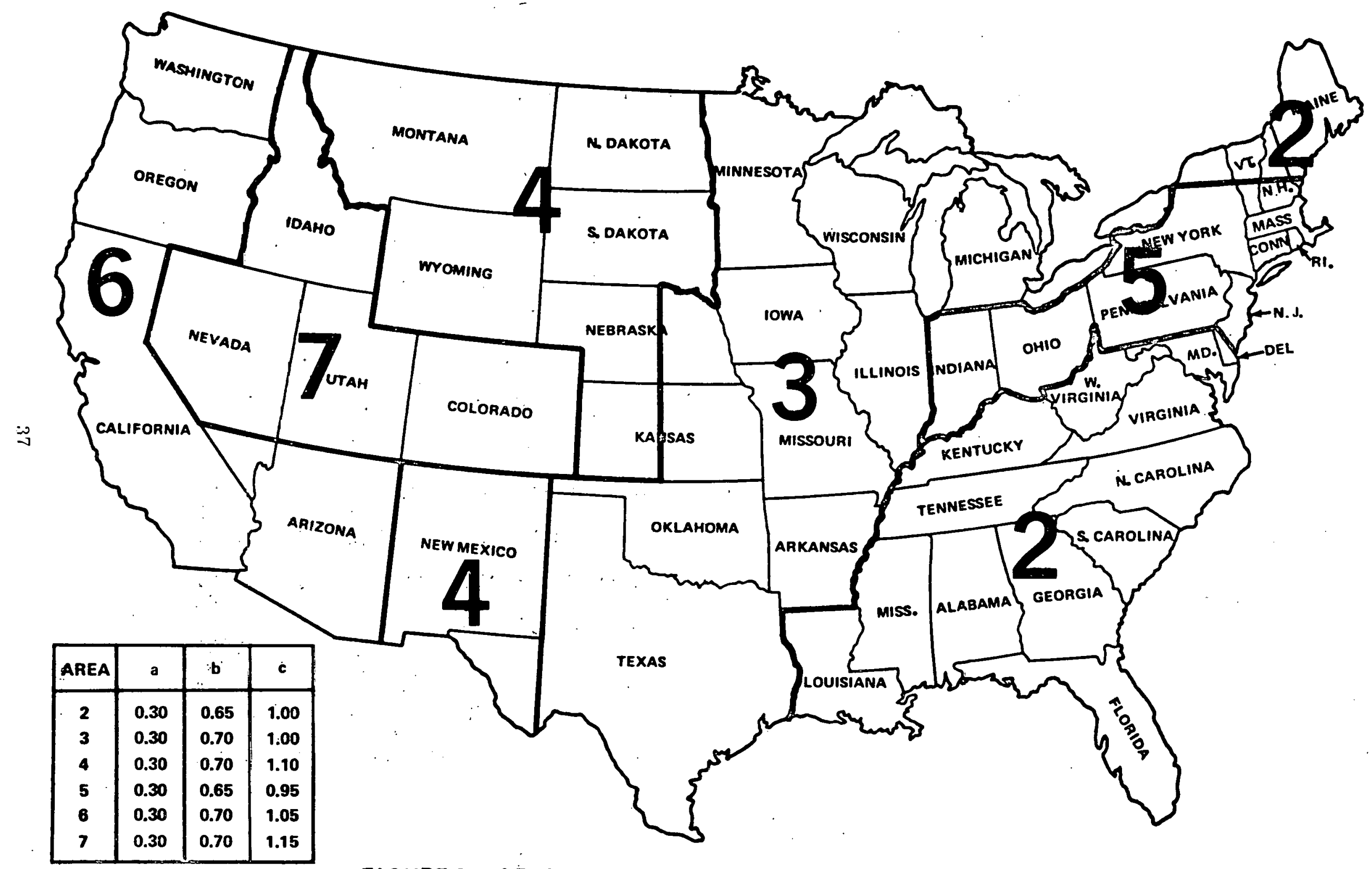

FIGURE 1. AREAS OF CONSTANT COEFFICIENTS IN EQUATION (6). 


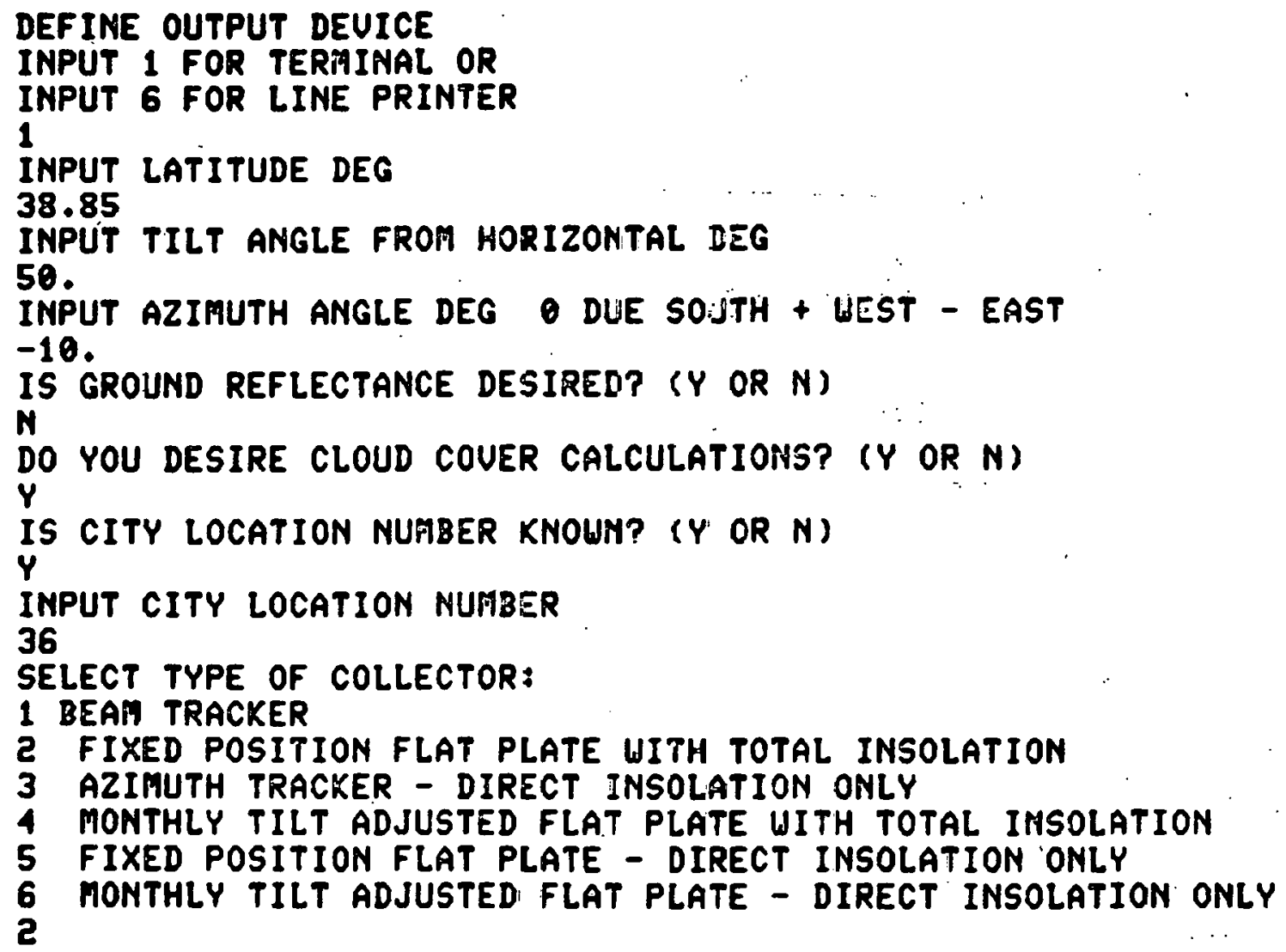

FIGURE 2. SAMPLE INPUT FOR ASHMET (FROM REMOTE TERMINAL) 


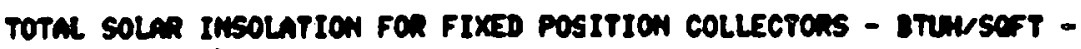

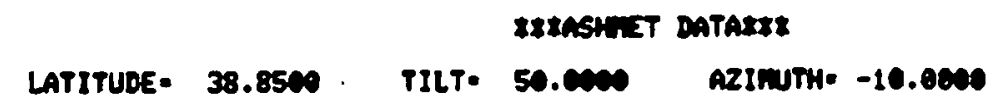

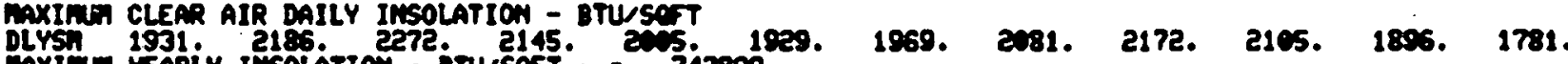

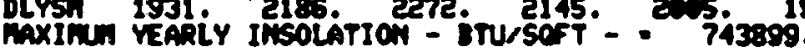

PROMABLE DAILY. IMSOLATION DUE TO CLOUD COUER

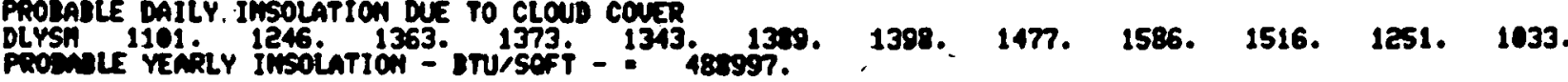

48.997. 


$$
\begin{gathered}
\text { APPROVAL } \\
\text { ASHMET - A COMPUTER CODE FOR ESTIMATING INSOLATION } \\
\text { INCIDENT ON TILTED SURFACES } \\
\text { - BY Robert F. E1kin and Ronald G. Toelle }
\end{gathered}
$$

The information in this report has been reviewed for technical content. Review of any information concerning Department of Defense or nuclear energy activities or programs has been made by the MSFC Security Classification officer. This report, in its entirety; has been determined to be unclassified.

TPbace

T. P. Isbell

Director, Systems Analysis and Integration Laboratory

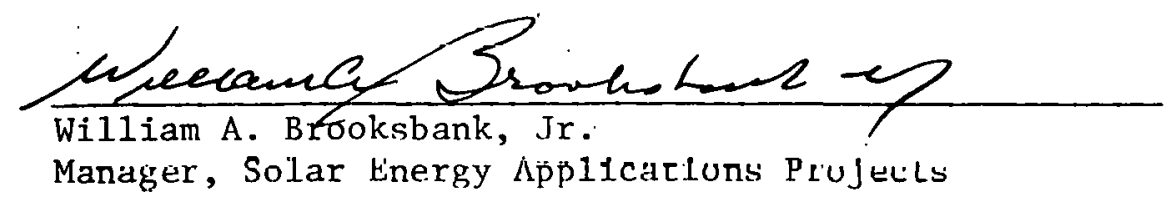

